\title{
Quantification of uncertainty in the prediction of railway induced ground vibration due to the use of statistical track unevenness data
}

\author{
Geert Lombaert $^{*, a}$, Pedro Galvín ${ }^{\mathrm{b}}$, Stijn François ${ }^{\mathrm{a}}$, Geert Degrande ${ }^{\mathrm{a}}$ \\ ${ }^{a} K U$ Leuven, Department of Civil Engineering, Kasteelpark Arenberg 40, B-3001 Leuven, Belgium \\ ${ }^{b}$ Universidad de Sevilla, Escuela Técnica Superior de Ingeniería, Camino de los Descubrimientos, 41092 Sevilla, Spain
}

\begin{abstract}
Environmental vibrations due to railway traffic are predominantly due to dynamic axle loads caused by wheel and track unevenness and impact excitation by rail joints and wheel flats. Because of its irregular character, track unevenness is commonly processed statistically and represented by its power spectral density function or its root mean square (RMS) value in one-third octave bands. This statistical description does not uniquely define the track unevenness at a given site, however, and different track unevenness profiles matching the statistical description will lead to different predictions of dynamic axle loads and resulting ground vibration. This paper presents a methodology that allows quantifying the corresponding variability in ground vibration predictions. The procedure is derived assuming the geometry of the track and soil to be homogeneous along the track. The procedure is verified by means of Monte Carlo simulations and its usefulness for assessing the mismatch between predicted and measured ground vibration is demonstrated in a case study. The results show that the response in time domain and its narrow band spectrum exhibit significant variability which is reduced when the running RMS value or the one-third octave band spectrum of the response are considered.
\end{abstract}

Key words: track unevenness, ground vibrations, dynamic vehicle-track interaction, random vibration, dynamic track-soil interaction, wave propagation

\section{Introduction}

Railway-induced ground vibration is generated by quasi-static and dynamic axle loads; the latter are due to several mechanisms such as wheel and rail unevenness, impact excitation due to rail joints and wheel flats, and parametric excitation due to sleeper periodicity [1]. These loads are transferred to the track, its supporting structure (ballast, subgrade, slab or tunnel) and the soil, where vibrations propagate as elastic waves and excite the foundations of nearby buildings.

Several numerical models have been recently developed for the prediction of railway induced ground vibration. Most of the modelling and computational effort usually goes to capturing the dynamic behavior of the track and the soil. The practical use of 3D finite element (FE) and finite element-boundary element (FE-BE) models is limited due to their high computational cost. Alternatively, dedicated models have been developed that exploit the regularity of the track and the underlying soil. When the track and the soil are assumed homogeneous in the direction along the track, a Fourier transformation of the coordinate along the track leads to an efficient solution in the frequency-wavenumber domain [2]. In this 2.5D methodology, a problem with a 2D geometry is solved for each frequency and wavenumber and the 3D solution is recovered by an inverse Fourier transformation. Because of their high computational efficiency and relatively modest

\footnotetext{
*Corresponding author. Phone: + 3216321772 .

Email address: geert. lombaert@bwk.kuleuven.be (Geert Lombaert)
} 
modeling effort, 2.5D methods have been applied by a large number of researchers to study dynamic traintrack interaction [3] as well as ground-borne vibration due to railway traffic at grade [4-8] and in tunnels $[9-12]$.

Track unevenness is generally regarded as one of the most important sources of railway induced ground vibration and often the only excitation mechanism considered in addition to quasi-static excitation $[7,9,13$, 14]. Because of its irregular character, track unevenness is commonly processed statistically and represented by its power spectral density (PSD) function $[15,16]$ or its root mean square (RMS) value in one-third octave bands [1]. This has the additional advantage of accomodating the combination of unevenness data measured in different ranges of wavelengths, e.g. long wavelength data obtained by track recording cars $[1,17]$ and short wavelength data from measurement trolleys [1, 17]. Alternatively, track unevenness can be extrapolated beyond the measured wavelength range by fitting measured data to generic PSD functions derived from large sets of data [18].

The statistical information on track unevenness in the form of a narrow band power spectral density function or RMS values in one-third octave bands can be used in two ways. First, one could generate a sample or realization of track unevenness matching the statistical description, introduce the sample in the model and compute the resulting dynamic axle loads and ground vibration. Calculations assuming a stationary Gaussian random field model for the track unevenness have shown a significant variability in resulting predicted ground vibration levels [18]. A second approach consists of computing the second order statistical characteristics of the ground vibration response based on the PSD function of the track unevenness. When the track geometry and properties are homogeneous along the track, as assumed in the aforementioned 2.5D models, the time history of each dynamic axle load is a stationary random process as well and the corresponding PSD function is calculated by random vibration theory [19]. The response at a fixed point on the track or in the free field, however, is a non-stationary random process due to the time-dependent relative position of the source and the receiver. Dedicated solution procedures $[13,18,20]$ have been developed that typically allow computing the mean square response, i.e. the average over different realizations of track unevenness. In the following, this is referred to as an ensemble average value.

Because of the significant scatter observed in predicted ground vibration levels for different realizations of track unevenness [18], it is important to quantify the response variability. In the following, a methodology is outlined that allows characterizing the scatter due to the use of statistical track unevenness data. Exact confidence bounds are derived for the mean square value of the response in the time and frequency domain and approximate confidence bounds are given for processed response quantities such as running root mean square (RMS) values and one-third octave band spectra which are often considered for the evaluation of environmental vibration. The method is derived assuming (1) a Gaussian random field model for the track unevenness and (2) that the geometry and properties of the track and soil are homogeneous along the track.

The outline of this paper is as follows. First, in section 2, the calculation of the response to a set of moving loads is briefly recapitulated. In section 3, the second-order statistics of the response are considered and the ensemble average response is calculated. Based on the derivation of the fourth-order statistics, the variability of the response is determined in section 4 . The case study presented in section 5 is considered for the verification of the methodology by Monte Carlo simulations in section 6. Finally, it is illustrated in section 7 how the quantification of uncertainty due to the use of statistical track unevenness data helps assessing the mismatch between measured and predicted ground vibration.

\section{Response due to moving loads}

\subsection{General formulation}

A brief recapitulation is made of the calculation of the response to a train which is travelling at a constant speed $v$ on a straight track. A right-handed Cartesian frame of reference $\mathbf{x}=(x, y, z)$ is considered with its origin at the centre of the track, the $y$-axis in the direction along the track, and the $z$-axis pointing upwards. The position of the axles therefore depends on the time $t$ as $\mathbf{x}_{k}(t)=\mathbf{x}_{k 0}+v t \mathbf{e}_{y}$ where $\mathbf{x}_{k 0}=\left\{x_{0}, y_{k 0}, z_{0}\right\}^{\mathrm{T}}$ is the position at $t=0$. In the case where the geometry and properties of the track 
and the soil are homogeneous in the direction along the track, the following expression for the displacement $\mathbf{u}(\mathbf{x}, t)$ at a point $\mathbf{x}=\{x, y, z\}^{\mathrm{T}}$ is derived by means of the Betti-Rayleigh reciprocity theorem [21]:

$$
\mathbf{u}(x, y, z, t)=\sum_{k=1}^{n_{\mathrm{a}}} \int_{-\infty}^{t} \mathbf{H}^{\mathrm{T}}\left(x, y-y_{k 0}-v \tau, z, t-\tau\right) \mathbf{g}_{k}(\tau) \mathrm{d} \tau
$$

where $n_{\mathrm{a}}$ is the total number of axles of the train, $\mathbf{g}_{k}(t)$ is the time history of the load applied to the track by the $k$-th axle and $\mathbf{H}^{\mathrm{T}}(x, y, z, t)$ is the $3 \times 3$ transfer function matrix. The three columns of the matrix $\mathbf{H}(x, y, z, t)$ represent the displacement at a point $\{x, y, z\}^{\mathrm{T}}$ for an impulse load applied at the point $\left\{x_{0}, 0, z_{0}\right\}^{\mathrm{T}}$ in the $x-, y-$, and $z$-direction, respectively. For convenience, the receiver coordinates $x$ and $z$ are omitted in the following.

\subsection{Response in frequency-wavenumber domain}

When the track geometry and properties are assumed homogeneous along the track, the transfer function can efficiently be computed in the frequency-wavenumber domain [22]. An expression for the response in the frequency-wavenumber domain is obtained by applying first a Fourier transform with respect to the time $t$ and next a Fourier transform with respect to the coordinate $y$. The Fourier transform pairs are defined as follows:

$$
\begin{aligned}
\hat{\mathbf{u}}(y, \omega) & =\int_{-\infty}^{+\infty} \mathbf{u}(y, t) \exp (-\mathrm{i} \omega t) \mathrm{d} t \\
\mathbf{u}(y, t) & =\frac{1}{2 \pi} \int_{-\infty}^{+\infty} \hat{\mathbf{u}}(y, \omega) \exp (\mathrm{i} \omega t) \mathrm{d} \omega
\end{aligned}
$$

and

$$
\begin{aligned}
\tilde{\mathbf{u}}\left(k_{y}, \omega\right) & =\int_{-\infty}^{+\infty} \hat{\mathbf{u}}(y, \omega) \exp \left(\mathrm{i} k_{y} y\right) \mathrm{d} y \\
\hat{\mathbf{u}}(y, \omega) & =\frac{1}{2 \pi} \int_{-\infty}^{+\infty} \tilde{\mathbf{u}}\left(k_{y}, \omega\right) \exp \left(-\mathrm{i} k_{y} y\right) \mathrm{d} k_{y}
\end{aligned}
$$

where a hat above a variable denotes its representation in the frequency-space domain and a tilde its representation in the frequency-wavenumber domain. Transformation of equation (1) to the frequencywavenumber domain gives:

$$
\tilde{\mathbf{u}}\left(k_{y}, \omega\right)=\sum_{k=1}^{n_{\mathrm{a}}} \tilde{\mathbf{H}}^{\mathrm{T}}\left(k_{y}, \omega\right) \hat{\mathbf{g}}_{k}\left(\omega-k_{y} v\right) \exp \left(+\mathrm{i} k_{y} y_{k 0}\right)
$$

The inverse wavenumber transform yields:

$$
\hat{\mathbf{u}}(y, \omega)=\sum_{k=1}^{n_{\mathrm{a}}} \frac{1}{2 \pi} \int_{-\infty}^{+\infty} \tilde{\mathbf{H}}^{\mathrm{T}}\left(k_{y}, \omega\right) \hat{\mathbf{g}}_{k}\left(\omega-k_{y} v\right) \exp \left[-\mathrm{i} k_{y}\left(y-y_{k 0}\right)\right] \mathrm{d} k_{y}
$$

A change of variables $\tilde{\omega}=\omega-k_{y} v$ gives the following expression:

$$
\hat{\mathbf{u}}(y, \omega)=\sum_{k=1}^{n_{\mathrm{a}}} \frac{1}{2 \pi} \int_{-\infty}^{+\infty} \underbrace{\left[\frac{1}{v} \tilde{\mathbf{H}}^{\mathrm{T}}\left(\frac{\omega-\tilde{\omega}}{v}, \omega\right) \exp \left[-\mathrm{i}\left(\frac{\omega-\tilde{\omega}}{v}\right)\left(y-y_{k 0}\right)\right]\right]}_{\hat{\mathbf{U}}^{\mathrm{T}}\left(y-y_{k 0}, \omega, \tilde{\omega}\right)} \hat{\mathbf{g}}_{k}(\tilde{\omega}) \mathrm{d} \tilde{\omega}
$$

The term between the brackets is the transponse of a matrix, denoted $\hat{\mathbf{U}}\left(y-y_{k 0}, \omega, \tilde{\omega}\right)$ of which the three columns represent the Fourier transform of the displacement at a point $\left\{x, y-y_{k 0}, z\right\}^{\mathrm{T}}$ for a moving load with 
time dependent position $\left\{x_{0}, v t, z_{0}\right\}^{\mathrm{T}}$ and an intensity $\exp \left(i \tilde{\omega}_{0} t\right)$, acting in the $x-, y-$, and $z$-direction, respectively. This can be verified by introducing the Fourier transform $2 \pi \delta\left(\tilde{\omega}-\tilde{\omega}_{0}\right)$ of this load in equation (8). Equation (8) is now rewritten as:

$$
\hat{\mathbf{u}}(y, \omega)=\sum_{k=1}^{n_{\mathrm{a}}} \frac{1}{2 \pi} \int_{-\infty}^{+\infty} \hat{\mathbf{U}}^{\mathrm{T}}\left(y-y_{k 0}, \omega, \tilde{\omega}\right) \hat{\mathbf{g}}_{k}(\tilde{\omega}) \mathrm{d} \tilde{\omega}
$$

The inverse Fourier transform with respect to $\omega$ allows computing the response $\mathbf{u}(y, t)$ in the time domain:

$$
\mathbf{u}(y, t)=\sum_{k=1}^{n_{\mathrm{a}}} \frac{1}{2 \pi} \int_{-\infty}^{+\infty} \mathbf{U}^{\mathrm{T}}\left(y-y_{k 0}, t, \tilde{\omega}\right) \hat{\mathbf{g}}_{k}(\tilde{\omega}) \mathrm{d} \tilde{\omega}
$$

where $\mathbf{U}^{\mathrm{T}}\left(y-y_{k 0}, t, \tilde{\omega}\right)$ represents the inverse Fourier transform of $\hat{\mathbf{U}}^{\mathrm{T}}\left(y-y_{k 0}, \omega, \tilde{\omega}\right)$.

\subsection{Decomposition into quasi-static and dynamic response contribution}

The axle load $\mathbf{g}_{k}(t)$ is now decomposed into a static component $\mathbf{g}_{\mathrm{s} k}$ and a dynamic component $\mathbf{g}_{\mathrm{d} k}(t)$. The static component $\mathbf{g}_{\mathrm{s} k}$ is determined by the weight $w_{k}$ carried by the axle and equals $-w_{k} \mathbf{e}_{z}$. The Fourier transform $\hat{\mathbf{g}}_{\mathrm{s} k}(\tilde{\omega})$ of the static load component is equal to $-w_{k} \mathbf{e}_{z} 2 \pi \delta(\tilde{\omega})$. The dynamic component is determined by several excitation mechanisms. The calculation of the dynamic load component is discussed extensively in the literature $[6,7,13]$ and therefore not repeated here. Introducing the decomposition $\hat{\mathbf{g}}_{k}(\tilde{\omega})=\hat{\mathbf{g}}_{\mathrm{s} k}(\tilde{\omega})+\hat{\mathbf{g}}_{\mathrm{d} k}(\tilde{\omega})=-w_{k} \mathbf{e}_{z} 2 \pi \delta(\tilde{\omega})+\hat{\mathbf{g}}_{\mathrm{d} k}(\tilde{\omega})$ in equation (9) allows distinguishing between the quasistatic contribution $\hat{\mathbf{u}}_{\mathrm{s}}(y, \omega)$ and dynamic contribution $\hat{\mathbf{u}}_{\mathrm{d}}(y, \omega)$ to the response $\hat{\mathbf{u}}(y, \omega)$ :

$$
\begin{aligned}
\hat{\mathbf{u}}(y, \omega) & =\hat{\mathbf{u}}_{\mathrm{s}}(y, \omega)+\hat{\mathbf{u}}_{\mathrm{d}}(y, \omega) \\
& =-\sum_{k=1}^{n_{\mathrm{a}}} w_{k} \hat{\mathbf{U}}^{\mathrm{T}}\left(y-y_{k 0}, \omega, 0\right) \mathbf{e}_{z}+\frac{1}{2 \pi} \int_{-\infty}^{+\infty} \hat{\mathbf{U}}^{\mathrm{T}}\left(y-y_{k 0}, \omega, \tilde{\omega}\right) \hat{\mathbf{g}}_{\mathrm{d} k}(\tilde{\omega}) \mathrm{d} \tilde{\omega}
\end{aligned}
$$

The quasi-static and dynamic contributions to the response in the time domain are found by introducing the decomposition $\hat{\mathbf{g}}_{k}(\tilde{\omega})=-w_{k} \mathbf{e}_{z} 2 \pi \delta(\tilde{\omega})+\hat{\mathbf{g}}_{\mathrm{d} k}(\tilde{\omega})$ in equation $(10)$ :

$$
\begin{aligned}
\mathbf{u}(y, t) & =\mathbf{u}_{\mathrm{s}}(y, t)+\mathbf{u}_{\mathrm{d}}(y, t) \\
& =-\sum_{k=1}^{n_{\mathrm{a}}} w_{k} \hat{\mathbf{U}}^{\mathrm{T}}\left(y-y_{k 0}, t, 0\right) \mathbf{e}_{z}+\frac{1}{2 \pi} \int_{-\infty}^{+\infty} \mathbf{U}^{\mathrm{T}}\left(y-y_{k 0}, t, \tilde{\omega}\right) \hat{\mathbf{g}}_{\mathrm{d} k}(\tilde{\omega}) \mathrm{d} \tilde{\omega}
\end{aligned}
$$

In the following, the case is considered where track unevenness, modelled as a stationary random field, is considered as excitation mechanism. Assuming linear behavior of the train and the track and a perfect contact between the wheels and the rails, the dynamic components $\mathbf{g}_{\mathrm{d} k}(t)$ of the axle loads depend linearly on the track unevenness [7] and represent a set of zero mean random processes. These random processes are mutually correlated as all axles experience the same track unevenness [18]. Because of the zero mean value of the dynamic load components, the mean value of the dynamic response $\mathbf{u}_{\mathrm{d}}(y, t)$ in equation (14) is zero as well and higher order statistics are required for its characterization. In the next section, the second-order statistical characteristics of the response are calculated, which allow for the computation of the RMS value of the response. This RMS value should be understood as the root of the mean square value of the response across different realizations of track unevenness. In order to characterize the scatter with respect to this ensemble average response value, the fourth-order statistical characteristics are considered next.

\section{Ensemble average of the absolute value of the response}

\subsection{Time domain response}

The ensemble average of the absolute value of the response in the time domain is determined by its second-order statistics as characterized by the non-stationary auto-correlation function (ACF):

$$
\left.\mathbf{R}\left(y, t_{1}, t_{2}\right)=\underset{4}{\langle\mathbf{u}}\left(y, t_{1}\right) \otimes \mathbf{u}\left(y, t_{2}\right)\right\rangle
$$


where $\otimes$ is the outer product and the brackets denote the ensemble average with respect to track unevenness realizations. The mean square value of the response at time $t$ is found by evaluating the ACF at $t=t_{1}=t_{2}$. By decomposing the total response according to equation (14), the ACF is elaborated as follows:

$$
\mathbf{R}\left(y, t_{1}, t_{2}\right)=\mathbf{u}_{\mathrm{s}}\left(y, t_{1}\right) \otimes \mathbf{u}_{\mathrm{s}}\left(y, t_{2}\right)+\left\langle\mathbf{u}_{\mathrm{d}}\left(y, t_{1}\right) \otimes \mathbf{u}_{\mathrm{d}}\left(y, t_{2}\right)\right\rangle
$$

where the outer products of the quasi-static and dynamic response contributions vanish due to the zero mean value of the dynamic response $\mathbf{u}_{\mathrm{d}}(y, t)$. The second term in equation (16) is the non-stationary ACF of the dynamic response denoted as $\mathbf{R}_{\mathrm{d}}\left(y, t_{1}, t_{2}\right)$ and computed following equation (14):

$$
\begin{aligned}
\mathbf{R}_{\mathrm{d}}\left(y, t_{1}, t_{2}\right)= & \left\langle\left[\sum_{k=1}^{n_{\mathrm{a}}} \frac{1}{2 \pi} \int_{-\infty}^{+\infty} \mathbf{U}^{\mathrm{T}}\left(y-y_{k 0}, t, \tilde{\omega}\right) \hat{\mathbf{g}}_{\mathrm{d} k}(\tilde{\omega}) \mathrm{d} \tilde{\omega}\right]\right. \\
& \left.\otimes\left[\sum_{l=1}^{n_{\mathrm{a}}} \frac{1}{2 \pi} \int_{-\infty}^{+\infty} \mathbf{U}^{\mathrm{T} *}\left(y-y_{l 0}, t, \tilde{\omega}^{\prime}\right) \hat{\mathbf{g}}_{\mathrm{d} l}^{*}\left(\tilde{\omega}^{\prime}\right) \mathrm{d} \tilde{\omega}^{\prime}\right]\right\rangle
\end{aligned}
$$

where the complex conjugate is denoted by the superscript star and a distinction is made between the variables $\tilde{\omega}$ and $\tilde{\omega}^{\prime}$ considered in the two integrations. Since the functions $\mathbf{U}^{\mathrm{T}}\left(y-y_{k 0}, t, \tilde{\omega}\right)$ and $\mathbf{U}^{\mathrm{T} *}(y-$ $\left.y_{l 0}, t, \tilde{\omega}^{\prime}\right)$ are deterministic, the ensemble average only affects the dynamic axle loads. Equation (17) is therefore rewritten as an inner product, denoted by the colon operator, leading to:

$$
\begin{aligned}
\mathbf{R}_{\mathrm{d}}\left(y, t_{1}, t_{2}\right)= & \sum_{k=1}^{n_{\mathrm{a}}} \sum_{l=1}^{n_{\mathrm{a}}} \frac{1}{4 \pi^{2}} \int_{-\infty}^{+\infty} \int_{-\infty}^{+\infty}\left[\mathbf{U}^{\mathrm{T}}\left(y-y_{k 0}, t, \tilde{\omega}\right) \otimes \mathbf{U}^{\mathrm{T} *}\left(y-y_{l 0}, t, \tilde{\omega}^{\prime}\right)\right] \\
& :\left\langle\hat{\mathbf{g}}_{\mathrm{d} k}(\tilde{\omega}) \otimes \hat{\mathbf{g}}_{\mathrm{d} l}^{*}\left(\tilde{\omega}^{\prime}\right)\right\rangle \mathrm{d} \tilde{\omega} \mathrm{d} \tilde{\omega}^{\prime}
\end{aligned}
$$

When the track geometry and properties are homogeneous along the track and the track unevenness is a stationary random field, the dynamic axle loads are stationary random processes and the following relation holds:

$$
\left\langle\hat{\mathbf{g}}_{\mathrm{d} k}(\tilde{\omega}) \otimes \hat{\mathbf{g}}_{\mathrm{d} l}^{*}\left(\tilde{\omega}^{\prime}\right)\right\rangle=4 \pi^{2} \hat{\mathbf{S}}_{\mathbf{g} k l}(\tilde{\omega}) \delta\left(\tilde{\omega}-\tilde{\omega}^{\prime}\right)
$$

where $\hat{\mathbf{S}}_{\mathbf{g} k l}(\tilde{\omega}) \delta\left(\tilde{\omega}^{\prime}-\omega\right)$ is the cross-power spectral density function of the dynamic axle loads $\hat{\mathbf{g}}_{\mathrm{d} k}(\tilde{\omega})$ and $\hat{\mathbf{g}}_{\mathrm{d} l}\left(\tilde{\omega}^{\prime}\right)$. The PSD functions of the dynamic axle loads can be calculated by random vibration theory [19], based on the expressions for the axle loads determined by means of a compliance formulation in a moving frame of reference [18]. The factor $4 \pi^{2}$ on the right hand side of equation (19) is due to the fact that, in the Fourier transform pair defined by equations (2) and (3), the factor $1 /(2 \pi)$ is applied to the inverse transform whereas for the relation between the ACF and PSD, the factor is in the forward transform in line with the usual convention. Introducing equation (19) in equation (18) leads to:

$$
\begin{aligned}
\mathbf{R}_{\mathrm{d}}\left(y, t_{1}, t_{2}\right)= & \sum_{k=1}^{n_{\mathrm{a}}} \sum_{l=1}^{n_{\mathrm{a}}} \int_{-\infty}^{+\infty}\left[\mathbf{U}^{\mathrm{T}}\left(y-y_{k 0}, t_{1}, \tilde{\omega}\right) \otimes \mathbf{U}^{\mathrm{T} *}\left(y-y_{l 0}, t_{2}, \tilde{\omega}\right)\right] \\
& : \hat{\mathbf{S}}_{\mathbf{g} k l}(\tilde{\omega}) \mathrm{d} \tilde{\omega}
\end{aligned}
$$

The cross terms $k \neq l$ on the right hand side of the equation represent the contributions due to crosscorrelation between the dynamic loads. When interaction through the vehicle and the track is disregarded and all axles have similar characteristics, the cross-power spectral density functions $\hat{\mathbf{S}}_{\mathbf{g} k l}(\tilde{\omega})$ have the same amplitude as the auto-power spectral density functions $\hat{\mathbf{S}}_{\mathbf{g} k k}(\tilde{\omega})$, but a different phase corresponding to the time lag between the excitation of different axles due to track unevenness.

The mean square response is obtained by evaluating the ACFs in equations (16) and (20) at $t=t_{1}=t_{2}$. Assuming that only vertical dynamic loads are applied to the track and that the only response quantity of 
interest is the vertical displacement, the total mean square response is computed from equations (16) and (20) as:

$$
\begin{aligned}
<u_{z}^{2}(y, t)> & =u_{\mathrm{s} z}^{2}(y, t)+R_{\mathrm{d} z z}(y, t, t) \\
& =u_{\mathrm{s} z}^{2}(y, t)+\sum_{k=1}^{n_{\mathrm{a}}} \sum_{l=1}^{n_{\mathrm{a}}} \int_{-\infty}^{+\infty} U_{z z}\left(y-y_{k 0}, t, \tilde{\omega}\right) U_{z z}^{*}\left(y-y_{l 0}, t, \tilde{\omega}\right) \hat{S}_{\mathbf{g} k k}(\tilde{\omega}) \mathrm{d} \tilde{\omega}
\end{aligned}
$$

where equation (20) has been used to elaborate the dynamic response. The time dependent RMS value of the free field response $u^{\mathrm{RMS}}(y, t)$ is computed from the mean square response in equation $(21)$ as $\sqrt{<u_{z}^{2}(y, t)>}$. This RMS value is an ensemble average at a particular time $t$ for different realizations of the dynamic axle loads or, equivalently, the track unevenness. For assessing vibration levels in case of transient vibration, use is often made of running RMS values on a finite time window, as recommended in the ISO 2631 standard [23], for example. Note that, although displacements are considered as response quantities in the present section, the results are easily generalized to velocities and accelerations. The running RMS value $u_{\mathrm{T}_{\mathrm{w}}}^{\mathrm{RMS}}(y, t)$ on a time window $T_{\mathrm{w}}$ is computed from the instantaneous RMS value as:

$$
u_{\mathrm{T}_{\mathrm{w}}}^{\mathrm{RMS}}(y, t)=\sqrt{\frac{1}{T_{\mathrm{w}}} \int_{t-T_{\mathrm{w}}}^{t}<u_{z}^{2}(y, \tau)>\mathrm{d} \tau}
$$

For a single realization, a running RMS value can be computed in a similar way by replacing the ensemble average value $\left\langle u_{z}^{2}(y, \tau)\right\rangle$ by the sample value $u_{z}^{2}(y, \tau)$ in equation $(22)$. Due to the non-stationarity of the response and the finite length of the time window considered, the running RMS value for this single realization will be different from the ensemble average. Since the integration in equation (22) is a linear operation, averaging the running mean square value for different realizations results in the same ensemble average running RMS value.

\subsection{Frequency domain response}

In the following, the ensemble average of the response in the frequency domain is computed based on the corresponding second-order statistics, i.e. the power spectrum of the response. Similarly to the ACF, the power spectrum $\hat{\mathbf{S}}\left(y, \omega_{1}, \omega_{2}\right)\left[\mathrm{m}^{2} /(\mathrm{rad} / \mathrm{s})^{2}\right]$ is decomposed in a quasi-static and dynamic contribution:

$$
\hat{\mathbf{S}}\left(y, \omega_{1}, \omega_{2}\right)=\hat{\mathbf{u}}_{\mathrm{s}}\left(y, \omega_{1}\right) \otimes \hat{\mathbf{u}}_{\mathrm{s}}^{*}\left(y, \omega_{2}\right)+\left\langle\hat{\mathbf{u}}_{\mathrm{d}}\left(y, \omega_{1}\right) \otimes \hat{\mathbf{u}}_{\mathrm{d}}^{*}\left(y, \omega_{2}\right)\right\rangle
$$

where again the outer product of quasi-static and dynamic response contributions vanishes. A similar decomposition has been used by Sheng et al. [13] for calculating the total power spectrum of vibrations by running trains. The second term in equation (23) is the power spectrum of the dynamic response, denoted as $\mathbf{S}_{\mathrm{d}}\left(y, \omega_{1}, \omega_{2}\right)\left[\mathrm{m}^{2} /(\mathrm{rad} / \mathrm{s})^{2}\right]$ and computed based on equation (12):

$$
\hat{\mathbf{S}}_{\mathrm{d}}\left(y, \omega_{1}, \omega_{2}\right)=\int_{-\infty}^{+\infty} \sum_{k=1}^{n_{\mathrm{a}}} \sum_{l=1}^{n_{\mathrm{a}}}\left[\hat{\mathbf{U}}^{\mathrm{T}}\left(y-y_{k 0}, \omega_{1}, \tilde{\omega}\right) \otimes \hat{\mathbf{U}}^{\mathrm{T} *}\left(y-y_{l 0}, \omega_{2}, \tilde{\omega}\right)\right]: \hat{\mathbf{S}}_{\mathbf{g} k l}(\tilde{\omega}) \mathrm{d} \tilde{\omega}
$$

As all train axles experience the same track unevenness, the resulting contributions to the free field response are usually similar apart from a small shift in time. This repeated passage of axles leads to the characteristic peaks and troughs in the narrow band frequency spectrum of the response that are determined by the axle and bogie passage frequencies [6, 24, 25]. Information on the time lag between the dynamic axle loads is present in the cross-power spectral density functions of the axle loads. If these cross terms are omitted in equation (24), the response in the frequency domain is smoothed [26]. A similar smoothing is obtained when the narrow band spectrum of the response in equation (23) is used to compute the power of the signal in one-third octave or octave bands [27, 28].

The total mean square response in the frequency domain is found by evaluating the power spectra in equations (23) and (24) at $\omega=\omega_{1}=\omega_{2}$. When only vertical dynamic loads and vertical response components 
are considered, the mean square response becomes:

$$
\begin{aligned}
<\left|\hat{u}_{z}(y, \omega)\right|^{2}> & =\left|\hat{u}_{\mathrm{s} z}(y, \omega)\right|^{2}+\hat{S}_{\mathrm{d} z z}(y, \omega, \omega) \\
& =\left|\hat{u}_{\mathrm{s} z}(y, \omega)\right|^{2}+\int_{-\infty}^{+\infty} \sum_{k=1}^{n_{\mathrm{a}}} \sum_{l=1}^{n_{\mathrm{a}}}\left[\hat{U}_{z z}\left(y-y_{k 0}, \omega, \tilde{\omega}\right) \otimes \hat{U}_{z z}^{*}\left(y-y_{l 0}, \omega, \tilde{\omega}\right)\right]: \hat{S}_{\mathrm{g} k l}(\tilde{\omega}) \mathrm{d} \tilde{\omega}
\end{aligned}
$$

Limit values for building vibration are often formulated in terms of one-third octave band spectra, e.g. for assessing vibration annoyance [29] or when considering the operation of vibration sensitive equipment [30, 31]. When integrating the narrow band spectra in equation (25), one should take into account that the mean square response $<\left|\hat{u}_{z}^{2}(y, \omega)\right|>$ depends on the length of the time window $T$ considered in the calculation of the response $\hat{U}_{z z}\left(y-y_{k 0}, \omega, \tilde{\omega}\right)$ due to a single load with harmonic time-varying intensity. Although the passage of a single load is a non-stationary event that does not allow for a meaningful quantitative assessment of RMS values in one-third octave bands, the passage of multiple train axles leads to a nearly stationary response signal. A quantitative analysis in one-third octave bands is therefore possible by normalizing the RMS values by the passage time $T_{\mathrm{p}}$ :

$$
v_{\left[\omega_{1}, \omega_{\mathrm{u}}\right]}^{\mathrm{RMS}}(y)=\sqrt{\frac{T_{\mathrm{p}}}{T}} \int_{\omega_{1}}^{\omega_{\mathrm{u}}} \sqrt{\hat{S}_{\mathrm{d} z z}(y, \omega, \omega)} \mathrm{d} \omega
$$

where $\omega_{\mathrm{l}}$ and $\omega_{\mathrm{u}}$ denote the lower and upper bound of the one-third octave band, respectively. The passage time $T_{\mathrm{p}}$ can be estimated based on the running RMS value of the total response as computed by equation (21), e.g. using the procedure for computation of one-third octave band spectra in the German standard DIN 45672-2 [32]. Note that by applying equation (26) all energy of the signal in the time window $T$ is moved into a signal with smaller duration $T_{\mathrm{p}}$. This results in a slight overestimation of the running RMS value of the signal contained in the passage time $T_{\mathrm{p}}$.

\section{Scatter of the response with respect to the ensemble average}

\subsection{Time domain response}

In the previous section, expressions have been derived that allow computing the mean square response which is an ensemble average with respect to track unevenness realizations. In order to verify the scatter of results across realizations of the track unevenness and resulting dynamic axle loads, a characterization of the fourth-order statistical moment is needed. In the following, a general expression is first derived and subsequently elaborated to provide the fourth-order statistical moment of the response at a particular time $t$ and the running RMS-value of the response on a fixed time window $T_{\mathrm{w}}$. It is explained how these results allow calculating confidence bounds that characterize the variability in the ground vibration predictions.

The fourth-order statistical moment of the free field response is given by the following fourth-order tensor:

$$
\mathbb{Q}\left(y, t_{1}, t_{2}, t_{3}, t_{4}\right)=\left\langle\mathbf{u}\left(y, t_{1}\right) \otimes \mathbf{u}\left(y, t_{2}\right) \otimes \mathbf{u}\left(y, t_{3}\right) \otimes \mathbf{u}\left(y, t_{4}\right)\right\rangle
$$

By decomposing the total response according to equation (14), the fourth-order statistical moment is elaborated as follows:

$$
\begin{aligned}
\mathbb{Q}\left(y, t_{1}, t_{2}, t_{3}, t_{4}\right) & =\left\langle\mathbf{u}_{\mathrm{s}}\left(y, t_{1}\right) \otimes \mathbf{u}_{\mathrm{s}}\left(y, t_{2}\right) \otimes \mathbf{u}_{\mathrm{s}}\left(y, t_{3}\right) \otimes \mathbf{u}_{\mathrm{s}}\left(y, t_{4}\right)\right\rangle \\
+ & \left\langle\mathbf{u}_{\mathrm{s}}\left(y, t_{1}\right) \otimes \mathbf{u}_{\mathrm{s}}\left(y, t_{2}\right) \otimes \mathbf{u}_{\mathrm{d}}\left(y, t_{3}\right) \otimes \mathbf{u}_{\mathrm{d}}\left(y, t_{4}\right)\right\rangle \\
+ & \left\langle\mathbf{u}_{\mathrm{s}}\left(y, t_{1}\right) \otimes \mathbf{u}_{\mathrm{d}}\left(y, t_{2}\right) \otimes \mathbf{u}_{\mathrm{s}}\left(y, t_{3}\right) \otimes \mathbf{u}_{\mathrm{d}}\left(y, t_{4}\right)\right\rangle \\
& +\left\langle\mathbf{u}_{\mathrm{s}}\left(y, t_{1}\right) \otimes \mathbf{u}_{\mathrm{d}}\left(y, t_{2}\right) \otimes \mathbf{u}_{\mathrm{d}}\left(y, t_{3}\right) \otimes \mathbf{u}_{\mathrm{s}}\left(y, t_{4}\right)\right\rangle \\
& +\left\langle\mathbf{u}_{\mathrm{d}}\left(y, t_{1}\right) \otimes \mathbf{u}_{\mathrm{s}}\left(y, t_{2}\right) \otimes \mathbf{u}_{\mathrm{s}}\left(y, t_{3}\right) \otimes \mathbf{u}_{\mathrm{d}}\left(y, t_{4}\right)\right\rangle \\
& +\left\langle\mathbf{u}_{\mathrm{d}}\left(y, t_{1}\right) \otimes \mathbf{u}_{\mathrm{s}}\left(y, t_{2}\right) \otimes \mathbf{u}_{\mathrm{d}}\left(y, t_{3}\right) \otimes \mathbf{u}_{\mathrm{s}}\left(y, t_{4}\right)\right\rangle \\
+ & \left\langle\mathbf{u}_{\mathrm{d}}\left(y, t_{1}\right) \otimes \mathbf{u}_{\mathrm{d}}\left(y, t_{2}\right) \otimes \mathbf{u}_{\mathrm{s}}\left(y, t_{3}\right) \otimes \mathbf{u}_{\mathrm{s}}\left(y, t_{4}\right)\right\rangle \\
+ & \left\langle\mathbf{u}_{\mathrm{d}}\left(y, t_{1}\right) \otimes \mathbf{u}_{\mathrm{d}}\left(y, t_{2}\right) \otimes \mathbf{u}_{\mathrm{d}}\left(y, t_{3}\right) \otimes \mathbf{u}_{\mathrm{d}}\left(y, t_{4}\right)\right\rangle \\
&
\end{aligned}
$$


where terms containing an odd number of dynamic response contributions have been omitted because of their zero mean value. Apart from the final term that contains the fourth-order statistical moment of the dynamic response $\mathbb{Q}_{\mathrm{d}}\left(y, t_{1}, t_{2}, t_{3}, t_{4}\right)$, all terms in equation (28) can be expressed in terms of the quasi-static response $\mathbf{u}_{\mathrm{s}}(y, t)$ and the non-stationary ACF of the dynamic response $\mathbf{R}_{\mathrm{d}}\left(y, t_{1}, t_{2}\right)$.

Equation (1) shows that the dynamic response is a linear transformation of the Gaussian random processes $\mathbf{g}_{\mathrm{d} k}(t)$ and, therefore, a Gaussian random process as well. In this case, Isserlis' theorem allows expressing the fourth-order moment of the dynamic response in terms of its second-order moments:

$$
\begin{aligned}
\mathbb{Q}_{\mathrm{d}}\left(y, t_{1}, t_{2}, t_{3}, t_{4}\right)= & \left\langle\mathbf{u}_{\mathrm{d}}\left(y, t_{1}\right) \otimes \mathbf{u}_{\mathrm{d}}\left(y, t_{2}\right) \otimes \mathbf{u}_{\mathrm{d}}\left(y, t_{3}\right) \otimes \mathbf{u}_{\mathrm{d}}\left(y, t_{4}\right)\right\rangle \\
= & \mathbf{R}_{\mathrm{d}}\left(y, t_{1}, t_{2}\right) \otimes \mathbf{R}_{\mathrm{d}}\left(y, t_{3}, t_{4}\right)+\mathbf{R}_{\mathrm{d}}\left(y, t_{1}, t_{3}\right) \otimes \mathbf{R}_{\mathrm{d}}\left(y, t_{2}, t_{4}\right) \\
& +\mathbf{R}_{\mathrm{d}}\left(y, t_{1}, t_{4}\right) \otimes \mathbf{R}_{\mathrm{d}}\left(y, t_{2}, t_{3}\right)
\end{aligned}
$$

Introducing equation (29) in equation (28), an expression for the fourth-order statistical moment of the response is obtained based on the previously derived solution in equation (20) for the non-stationary ACF $\mathbf{R}_{\mathrm{d}}\left(y, t_{1}, t_{2}\right)$. Note that the derivation of the latter only requires the assumption of stationarity for the dynamic axle loads, whereas equation (29) additionally requires the assumption of a Gaussian random process model.

As in the case of the non-stationary $\mathrm{ACF} \mathbf{R}_{\mathrm{d}}\left(y, t_{1}, t_{2}\right)$, the evaluation of the fourth-order statistical moment for the case where $t=t_{1}=t_{2}=t_{3}=t_{4}$ is of most interest, as it allows assessing the scatter of the response with respect to the RMS value. When applied for this particular case, equation (28) gives the following expression for the fourth-order moment of the vertical response:

$$
\begin{aligned}
\mathbb{Q}_{z z z z}(y, t, t, t, t) & =\left\langle u_{z}^{4}(y, t)\right\rangle \\
& =u_{\mathrm{s} z}^{4}(y, t)+6 u_{\mathrm{s} z}^{2}(y, t)\left\langle u_{\mathrm{d} z}^{2}(y, t)\right\rangle+\left\langle u_{\mathrm{d} z}^{4}(y, t)\right\rangle
\end{aligned}
$$

The last term on the right hand side of equation (30) is computed by applying equation (29) for the case where $t=t_{1}=t_{2}=t_{3}=t_{4}$ :

$$
\left\langle u_{\mathrm{d} z}^{4}(y, t)\right\rangle=3\left\langle u_{\mathrm{d} z}^{2}(y, t)\right\rangle^{2}
$$

The fourth-order moment $\left\langle u_{\mathrm{d} z}^{4}(y, t)\right\rangle$ characterizes the scatter of the square value of the dynamic response $u_{\mathrm{d} z}^{2}(y, t)$ with respect to the mean square value $\left\langle u_{\mathrm{d} z}^{2}(y, t)\right\rangle$. Since the dynamic response $u_{\mathrm{d} z}(y, t)$ at fixed position $y$ and time $t$ is a Gaussian random variable, its squared value, divided by its mean square value follows a chi-square distribution with a single degree of freedom:

$$
\frac{u_{\mathrm{d} z}^{2}(y, t)}{\left\langle u_{\mathrm{d} z}^{2}(y, t)\right\rangle} \sim \chi^{2}(1)
$$

Confidence intervals for the squared dynamic response contribution can therefore be derived from the cumulative distribution function (CDF) of the chi-square distribution. For example, it follows from the values of the CDF for the $12.5 \%$ and $87.5 \%$ quantiles that $75 \%$ of the realizations for $u_{\mathrm{d} z}^{2}(y, t)$ lie between $\alpha=0.025$ and $\beta=2.35$ times the mean square value $\left\langle u_{\mathrm{d} z}^{2}(y, t)\right\rangle$. The corresponding absolute value of the vibration response $\left|u_{\mathrm{d} z}(y, t)\right|=\sqrt{u_{\mathrm{d} z}^{2}(y, t)}$ is between $\sqrt{\alpha}=0.16$ and $\sqrt{\beta}=1.53$ times the RMS value, indicating that a considerable degree of scatter is expected when the dynamic contribution dominates the total response.

The relation (31) between the fourth-order and second-order moment of the dynamic response is now used to compute the fourth-order statistical moment of the total response in equation (30):

$$
\begin{aligned}
\mathbb{Q}_{z z z z}(y, t, t, t, t) & =\left\langle u_{z}^{4}(y, t)\right\rangle \\
& =u_{\mathrm{s} z}^{4}(y, t)+6 u_{\mathrm{s} z}^{2}(y, t)\left\langle u_{\mathrm{d} z}^{2}(y, t)\right\rangle+3\left\langle u_{\mathrm{d} z}^{2}(y, t)\right\rangle^{2}
\end{aligned}
$$

Since the total response $u_{z}(y, t)$ has a non-zero mean value $u_{\mathrm{s} z}(y, t)$, the squared value of the total response, divided by the mean square value of the dynamic response follows a non-central chi-square distribution with 
a single degree of freedom:

$$
\frac{u_{z}^{2}(y, t)}{\left\langle u_{\mathrm{d} z}^{2}(y, t)\right\rangle} \sim \chi_{\mathrm{NC}}^{2}(1)
$$

The non-centrality parameter $\lambda$ of the non-central chi-square distribution depends on the mean value and variance of the total response:

$$
\lambda=\frac{\left\langle u_{z}(y, t)\right\rangle^{2}}{\left\langle u_{\mathrm{d} z}(y, t)\right\rangle^{2}}=\frac{u_{\mathrm{s} z}^{2}(y, t)}{\left\langle u_{\mathrm{d} z}(y, t)\right\rangle^{2}}
$$

For example, when the non-centrality parameter $\lambda=1$, i.e. when $\left\langle u_{z}(y, t)\right\rangle^{2}=\left\langle u_{\mathrm{d} z}(y, t)\right\rangle^{2}, 75 \%$ of the realizations for $u_{z}^{2}(y, t)$ lie between $\alpha=0.067$ and $\beta=4.64$ times the mean square value of the dynamic response contribution $\left\langle u_{\mathrm{d} z}^{2}(y, t)\right\rangle$ or $\alpha / 2=0.033$ and $\beta / 2=2.32$ times the mean square value of the total response $\left\langle u_{z}^{2}(y, t)\right\rangle$. The corresponding absolute value of the total response $\left|u_{z}(y, t)\right|=\sqrt{u_{z}^{2}(y, t)}$ is between $\sqrt{\alpha / 2}=0.18$ and $\sqrt{\beta / 2}=1.52$ times the RMS value $\sqrt{\left\langle u_{z}^{2}(y, t)\right\rangle}$, indicating that the degree of variability is slightly reduced when compared to the case where the dynamic contribution dominates the total response. When the static contribution dominates the total response, the non-centrality parameter $\lambda$ increases and the variability of the total response is smaller.

The aforementioned numbers only hold for the variability of the instantaneous response value $\sqrt{\left\langle u_{z}(y, t)\right\rangle^{2}}$. In order to assess the variability of the running RMS value of the response over a time window $T_{\mathrm{w}}$ in equation (22), the following averaged response quantity needs to be considered:

$$
\begin{aligned}
& \left\langle\left[u_{\mathrm{T}_{\mathrm{w}}}^{\mathrm{RMS}}\left(y, t_{1}\right)\right]^{2}\left[u_{\mathrm{T}_{\mathrm{w}}}^{\mathrm{RMS}}\left(y, t_{2}\right)\right]^{2}\right\rangle= \\
& \left\langle\left[\frac{1}{T_{\mathrm{w}}} \int_{t_{1}-T_{\mathrm{w}}}^{t_{1}} u_{z}^{2}\left(y, \tau_{1}\right) \mathrm{d} \tau_{1}\right]\left[\frac{1}{T_{\mathrm{w}}} \int_{t_{2}-T_{\mathrm{w}}}^{t_{2}} u_{z}^{2}\left(y, \tau_{2}\right) \mathrm{d} \tau_{2}\right]\right\rangle
\end{aligned}
$$

This expression is further elaborated for the particular case $t=t_{1}=t_{2}$ by decomposing the total response according to equation (14):

$$
\begin{aligned}
& \left\langle\left[u_{\mathrm{T}_{\mathrm{w}}}^{\mathrm{RMS}}(y, t)\right]^{4}\right\rangle=\frac{1}{T_{\mathrm{w}}^{2}} \int_{t-T_{\mathrm{w}}}^{t} \int_{t-T_{\mathrm{w}}}^{t}\left\langle u_{\mathrm{s} z}^{2}\left(y, \tau_{1}\right) u_{\mathrm{s} z}^{2}\left(y, \tau_{2}\right)\right. \\
& \quad+2 u_{\mathrm{s} z}^{2}\left(y, \tau_{1}\right) u_{\mathrm{s} z}\left(y, \tau_{2}\right) u_{\mathrm{d} z}\left(y, \tau_{2}\right)+u_{\mathrm{s} z}^{2}\left(y, \tau_{1}\right) u_{\mathrm{d} z}^{2}\left(y, \tau_{2}\right) \\
& \quad+2 u_{\mathrm{s} z}\left(y, \tau_{1}\right) u_{\mathrm{d} z}\left(y, \tau_{1}\right) u_{\mathrm{s} z}^{2}\left(y, \tau_{2}\right)+4 u_{\mathrm{s} z}\left(y, \tau_{1}\right) u_{\mathrm{d} z}\left(y, \tau_{1}\right) u_{\mathrm{s} z}\left(y, \tau_{2}\right) u_{\mathrm{d} z}\left(y, \tau_{2}\right) \\
& \quad+2 u_{\mathrm{s} z}\left(y, \tau_{1}\right) u_{\mathrm{d} z}\left(y, \tau_{1}\right) u_{\mathrm{d} z}^{2}\left(y, \tau_{2}\right)+u_{\mathrm{d} z}^{2}\left(y, \tau_{1}\right) u_{\mathrm{s} z}^{2}\left(y, \tau_{2}\right) \\
& \left.\quad+2 u_{\mathrm{d} z}^{2}\left(y, \tau_{1}\right) u_{\mathrm{s} z}\left(y, \tau_{2}\right) u_{\mathrm{d} z}\left(y, \tau_{2}\right)+u_{\mathrm{d} z}^{2}\left(y, \tau_{1}\right) u_{\mathrm{d} z}^{2}\left(y, \tau_{2}\right)\right\rangle \mathrm{d} \tau_{1} \mathrm{~d} \tau_{2}
\end{aligned}
$$

The terms containing an odd number of the dynamic response contributions vanish and can therefore be omitted. By rewriting the average response quantities of the dynamic response in terms of the ACF and the fourth-order statistical moment, equation (37) becomes:

$$
\begin{aligned}
& \left\langle\left[u_{\mathrm{T}_{\mathrm{w}}}^{\mathrm{RMS}}(y, t)\right]^{4}\right\rangle=\left[u_{\mathrm{sT}}^{\mathrm{RMS}}(y, t)\right]^{4} \\
& \quad+2\left[u_{\mathrm{s} \mathrm{w}_{\mathrm{w}}}^{\mathrm{RMS}}(y, t)\right]^{2} \frac{1}{T_{\mathrm{w}}} \int_{t-T_{\mathrm{w}}}^{t} R_{\mathrm{d} z z}\left(y, \tau_{1}, \tau_{1}\right) \mathrm{d} \tau_{1} \\
& \quad+\frac{4}{T_{\mathrm{w}}^{2}} \int_{t-T_{\mathrm{w}}}^{t} \int_{t-T_{\mathrm{w}}}^{t} u_{\mathrm{s} z}\left(y, \tau_{1}\right) u_{\mathrm{s} z}\left(y, \tau_{2}\right) R_{\mathrm{d} z z}\left(y, \tau_{1}, \tau_{2}\right) \mathrm{d} \tau_{1} \mathrm{~d} \tau_{2} \\
& \quad+\frac{1}{T_{\mathrm{w}}^{2}} \int_{t-T_{\mathrm{w}}}^{t} \int_{t-T_{\mathrm{w}}}^{t} Q_{\mathrm{d} z z}\left(y, \tau_{1}, \tau_{1}, \tau_{2}, \tau_{2}\right) \mathrm{d} \tau_{1} \mathrm{~d} \tau_{2}
\end{aligned}
$$


where $u_{\mathrm{sT}_{\mathrm{w}}}^{\mathrm{RMS}}(y, t)$ represents the running RMS value on a time window $T_{w}$ of the static response contribution. By introducing expression (29) for the fourth-order moment of the dynamic response contribution $\mathbb{Q}_{\mathrm{d}}\left(y, t_{1}, t_{2}, t_{3}, t_{4}\right)$, equation (38) becomes:

$$
\begin{aligned}
& \left\langle\left[u_{\mathrm{T}_{\mathrm{w}}}^{\mathrm{RMS}}(y, t)\right]^{4}\right\rangle=\left[u_{\mathrm{s} \mathrm{w}_{\mathrm{w}}}^{\mathrm{RMS}}(y, t)\right]^{4} \\
& \quad+2\left[u_{\mathrm{s} \mathrm{w}_{\mathrm{w}}}^{\mathrm{RMS}}(y, t)\right]^{2} \frac{1}{T_{\mathrm{w}}} \int_{t-T_{\mathrm{w}}}^{t} R_{\mathrm{d} z z}\left(y, \tau_{1}, \tau_{1}\right) \mathrm{d} \tau_{1} \\
& \quad+\frac{4}{T_{\mathrm{w}}^{2}} \int_{t-T_{\mathrm{w}}}^{t} \int_{t-T_{\mathrm{w}}}^{t} u_{\mathrm{s} z}\left(y, \tau_{1}\right) u_{\mathrm{s} z}\left(y, \tau_{2}\right) R_{\mathrm{d} z z}\left(y, \tau_{1}, \tau_{2}\right) \mathrm{d} \tau_{1} \mathrm{~d} \tau_{2} \\
& \quad+\left[\frac{1}{T_{\mathrm{w}}} \int_{t-T_{\mathrm{w}}}^{t} R_{\mathrm{d} z z}\left(y, \tau_{1}, \tau_{1}\right) \mathrm{d} \tau_{1}\right]^{2}+\frac{2}{T_{\mathrm{w}}^{2}} \int_{t-T_{\mathrm{w}}}^{t} \int_{t-T_{\mathrm{w}}}^{t}\left[R_{\mathrm{d} z z}\left(y, \tau_{1}, \tau_{2}\right)\right]^{2} \mathrm{~d} \tau_{1} \mathrm{~d} \tau_{2}
\end{aligned}
$$

By denoting the running RMS value of the dynamic response on a time window $T_{w}$ as $u_{\mathrm{dT}}^{\mathrm{RMS}}(y, t)$, equation (39) becomes:

$$
\begin{gathered}
\left\langle\left[u_{\mathrm{T}_{\mathrm{w}}}^{\mathrm{RMS}}(y, t)\right]^{4}\right\rangle=\left[u_{\mathrm{s} \mathrm{w}_{\mathrm{w}}}^{\mathrm{RMS}}(y, t)\right]^{4}+2\left[u_{\mathrm{s} \mathrm{T}_{\mathrm{w}}}^{\mathrm{RMS}}(y, t)\right]^{2}\left[u_{\mathrm{d} \mathrm{T}_{\mathrm{w}}}^{\mathrm{RMS}}(y, t)\right]^{2} \\
+\frac{4}{T_{\mathrm{w}}^{2}} \int_{t-T_{\mathrm{w}}}^{t} \int_{t-T_{\mathrm{w}}}^{t} u_{\mathrm{s} z}\left(y, \tau_{1}\right) u_{\mathrm{s} z}\left(y, \tau_{2}\right) R_{\mathrm{d} z z}\left(y, \tau_{1}, \tau_{2}\right) \mathrm{d} \tau_{1} \mathrm{~d} \tau_{2} \\
+\left[u_{\mathrm{d} T_{\mathrm{w}}}^{\mathrm{RMS}}(y, t)\right]^{4}+\frac{2}{T_{\mathrm{w}}^{2}} \int_{t-T_{\mathrm{w}}}^{t} \int_{t-T_{\mathrm{w}}}^{t}\left[R_{\mathrm{d} z z}\left(y, \tau_{1}, \tau_{2}\right)\right]^{2} \mathrm{~d} \tau_{1} \mathrm{~d} \tau_{2}
\end{gathered}
$$

Equation (40) shows that the variability of the running RMS value on a time window $T_{\mathrm{w}}$ depends on the correlation of the dynamic response. In the limiting case $T_{\mathrm{w}} \rightarrow 0$, the dynamic response contributions are fully correlated on the time window $T_{w}$ and the $\operatorname{ACF} R_{\mathrm{d} z z}\left(y, \tau_{1}, \tau_{2}\right)$ for $t-T_{\mathrm{w}} \leq \tau_{1}, \tau_{2} \leq t$ can be approximated by the product of the standard deviations of the dynamic response contributions $u_{\mathrm{dT}}^{\mathrm{RMS}}\left(y, \tau_{1}\right) u_{\mathrm{dT}}^{\mathrm{RMS}}\left(y, \tau_{2}\right)$ with, additionally $\tau_{1}, \tau_{2} \rightarrow t$ as $T_{\mathrm{w}} \rightarrow 0$. Under the same limiting conditions, $u_{\mathrm{s} z}\left(y, \tau_{1}\right) \approx u_{\mathrm{s} z}(y, t)$ and the previously derived expression (33) for the instantaneous running RMS value is retrieved. Confidence bounds in this case are therefore found from the CDF of the non-central chi-square distribution with a single degree of freedom. If the correlation function decays rapidly in the time window $T_{\mathrm{w}}$ or, equivalently, if the correlation time $t_{\mathrm{c}}$ is small compared to $T_{\mathrm{w}}$, the integrals on the right hand side of equation (40) containing the $\operatorname{ACF} R_{\mathrm{d} z z}\left(y, \tau_{1}, \tau_{2}\right)$ can be disregarded:

$$
\begin{aligned}
\left\langle\left[u_{\mathrm{T}_{\mathrm{w}}}^{\mathrm{RMS}}(y, t)\right]^{4}\right\rangle \approx & {\left[u_{\mathrm{sT}_{\mathrm{w}}}^{\mathrm{RMS}}(y, t)\right]^{4}+2\left[u_{\mathrm{sT}_{\mathrm{w}}}^{\mathrm{RMS}}(y, t)\right]^{2}\left[u_{\mathrm{dT}_{\mathrm{w}}}^{\mathrm{RMS}}(y, t)\right]^{2} } \\
& +\left[u_{\mathrm{sT}_{\mathrm{w}}}^{\mathrm{RMS}}(y, t)\right]^{4}
\end{aligned}
$$

which is equal to the square of the total mean square response, showing that in this case the variability with respect to the mean square value vanishes.

The limiting cases (1) $T_{\mathrm{w}} \rightarrow 0$, with full correlation, i.e. $t_{\mathrm{c}}>>T_{\mathrm{w}}$, and $(2) t_{\mathrm{c}}<<T_{\mathrm{w}}$ show that integrating the running RMS value of the response over time as in equation (22) reduces the scatter about the ensemble average. A conservative estimation of the variability of the running RMS value on a time window $T_{\mathrm{w}}$ is obtained when the squared response is fully correlated over the time window $T_{\mathrm{w}}$, implying that the latter has the same variability as the original instantaneous running RMS value.

\subsection{Frequency domain response}

A similar derivation can now be made for the frequency domain representation of the total response. As in the case of the time domain response, most interest lies in the derivation of confidence bounds that allow verifying the scatter with respect to the mean square response in equation (25) and the frequency band RMS values in equation (26). 
The frequency domain response is a complex valued random process of which the real and imaginary part of the response follow the same Gaussian distribution with zero mean value. The modulus of the response is therefore the absolute value of a circular bivariate Gaussian random variable. The probability distribution of the latter follows the so-called Rayleigh distribution or, equivalently, the modulus of the dynamic response contribution, normalized by the squared standard deviation of the real and imaginary part, follows a chi-square distribution with two degrees of freedom:

$$
\frac{2\left[\operatorname{Re}\left\{\hat{u}_{\mathrm{d} z}(y, \omega)\right\}\right]^{2}}{\left\langle\left|\hat{u}_{\mathrm{d} z}(y, t)\right|^{2}\right\rangle}+\frac{2\left[\operatorname{Im}\left\{\hat{u}_{\mathrm{d} z}(y, \omega)\right\}\right]^{2}}{\left\langle\left|\hat{u}_{\mathrm{d} z}(y, t)\right|^{2}\right\rangle}=\frac{2\left|\hat{u}_{\mathrm{d} z}(y, \omega)\right|^{2}}{\left\langle\left|\hat{u}_{\mathrm{d} z}(y, t)\right|^{2}\right\rangle} \sim \chi^{2}(2)
$$

where the factors of two stem from the normalization based on the standard deviation of the real or imaginary part. From the CDF of the chi-square distribution with two degrees of freedom, it follows that $75 \%$ of the realizations for $\left|\hat{u}_{\mathrm{d} z}(y, \omega)\right|^{2}$ are between $\alpha=0.134$ and $\beta=2.08$ times the mean square value $\left\langle\left|\hat{u}_{\mathrm{d} z}(y, t)\right|^{2}\right\rangle$. The corresponding absolute value of the vibration response $\left|\hat{u}_{\mathrm{d} z}(y, \omega)\right|$ is between $\sqrt{\alpha}=0.37$ and $\sqrt{\beta}=1.44$ times the RMS response or $8.7 \mathrm{~dB}$ less and $3.2 \mathrm{~dB}$ more than the RMS response, respectively.

When the total response is considered, the real and imaginary part have a non-zero mean value determined by the static response contribution $\hat{u}_{\mathrm{s} z}(y, \omega)$. In this case, the squared modulus of the response, normalized by the mean square value of the dynamic response follows a non-central chi-square distribution with two degrees of freedom:

$$
\frac{2\left[\operatorname{Re}\left\{\hat{u}_{z}(y, \omega)\right\}\right]^{2}}{\left\langle\left|\hat{u}_{\mathrm{d} z}(y, \omega)\right|^{2}\right\rangle}+\frac{2\left[\operatorname{Im}\left\{\hat{u}_{z}(y, \omega)\right\}\right]^{2}}{\left\langle\left|\hat{u}_{\mathrm{d} z}(y, \omega)\right|^{2}\right\rangle}=\frac{2\left|\hat{u}_{z}(y, \omega)\right|^{2}}{\left\langle\left|\hat{u}_{\mathrm{d} z}(y, \omega)\right|^{2}\right\rangle} \sim \chi_{\mathrm{NC}}^{2}(2)
$$

The non-centrality parameter $\lambda$ of the non-central chi-square distribution depends on the mean value and variance of the real and imaginary part of the total response:

$$
\begin{aligned}
\lambda & =2 \frac{\left\langle\operatorname{Re}\left\{\hat{u}_{z}(y, \omega)\right\}\right\rangle^{2}}{\left.\left\langle\mid \hat{u}_{\mathrm{d} z}(y, \omega)\right\rangle\right|^{2}}+2 \frac{\left\langle\operatorname{Im}\left\{\hat{u}_{z}(y, \omega)\right\}\right\rangle^{2}}{\left.\left\langle\mid \hat{u}_{\mathrm{d} z}(y, \omega)\right\rangle\right|^{2}} \\
& =2 \frac{\left[\operatorname{Re}\left\{\hat{u}_{\mathrm{s} z}(y, \omega)\right\}\right]^{2}}{\left.\left\langle\mid \hat{u}_{\mathrm{d} z}(y, \omega)\right\rangle\right|^{2}}+2 \frac{\left[\operatorname{Im}\left\{\hat{u}_{\mathrm{s} z}(y, \omega)\right\}\right]^{2}}{\left.\left\langle\mid \hat{u}_{\mathrm{d} z}(y, \omega)\right\rangle\right|^{2}}=2 \frac{\left|\hat{u}_{\mathrm{s} z}(y, \omega)\right|^{2}}{\left\langle\left|u_{\mathrm{d} z}(y, \omega)\right|^{2}\right\rangle}
\end{aligned}
$$

When both response contributions have the same mean square value, i.e. $\left\langle\left|\hat{u}_{\mathrm{s} z}(y, \omega)\right|^{2}\right\rangle=\left\langle\left|\hat{u}_{\mathrm{d} z}(y, \omega)\right|^{2}\right\rangle$, the non-centrality parameter $\lambda=2$ and $75 \%$ of the realizations for $\left|\hat{u}_{z}(y, t)\right|^{2}$ lie between $\alpha=0.34$ and $\beta=3.98$ times the mean square value of the dynamic response contribution $\left\langle\left|\hat{u}_{\mathrm{d} z}(y, \omega)\right|^{2}\right\rangle$ or $\alpha / 2=0.17$ and $\beta / 2=1.99$ times the mean square value of the total response $\left\langle\left|\hat{u}_{z}(y, \omega)\right|^{2}\right\rangle$. The corresponding absolute value of the total response $\left|\hat{u}_{z}(y, \omega)\right|$ is between $\sqrt{\alpha / 2}=0.41$ and $\sqrt{\beta}=1.41$ times the RMS response or $7.7 \mathrm{~dB}$ less and $3.0 \mathrm{~dB}$ more than the RMS response, respectively.

The foregoing discussion only holds for the modulus of the response evaluated at a single frequency. The scatter of the response in one-third octave bands depends on the correlation in the frequency domain and, therefore, on the values of the power spectrum $\hat{\mathbf{S}}_{\mathrm{d}}\left(y, \omega_{1}, \omega_{2}\right)$ for the case where $\omega_{1} \neq \omega_{2}$. Equation (23) for the power spectrum shows that, due to the motion of the load, different response frequencies $\omega_{1}$ and $\omega_{2}$ are coupled to the same source frequency $\tilde{\omega}$, and, therefore, correlated. For a vanishing load speed $v \rightarrow 0$, the response at a fixed point becomes a stationary random process with a delta-correlated power spectrum as in the case of the dynamic axle loads in equation (19). A conservative estimation of the variability of the one-third octave band spectrum is obtained by assuming that the squared response is fully correlated over each one-third octave band, implying that the latter has the same variability as the original narrow band frequency spectrum. 


\section{Case study}

\subsection{Introduction}

The present section introduces a case study of ground vibrations at a site located at Saint-Epain (France) along the conventional line Paris-Bordeaux. A brief description is given of the dynamic characteristics of the track, soil, and rolling stock, as provided by SNCF (Société Nationale des Chemins de fer Français), and the corresponding numerical model is introduced. Next, in section 6 , the expressions that have been derived for calculating the ensemble average response quantities and their variability are verified. For this purpose, the results from these expressions are compared to estimations based on Monte Carlo simulations for a large number of track unevenness realizations. Finally, in section 7, the predicted and measured ground vibrations are compared to illustrate the usefulness of the proposed approach.

\subsection{The dynamic characteristics of the track and the soil}

A geological section determined from a boring close to the measurement site shows that the soil consists of a layer of sand and gravel with a thickness of about $2 \mathrm{~m}$, followed by siliceous formations with a thickness of about $10 \mathrm{~m}$ on top of chalk, the latter two formations dating from the late Cretaceous. The dynamic soil characteristics at the site have been identified by means of a Spectral Analysis of Surface Waves (SASW) test. The shear wave velocity profile was identified from the SASW test by fitting the computed dispersion curve to the experimental dispersion curve of the fundamental Rayleigh wave at the site (figure 1b). The results show that the soil can be represented by a shallow soft top layer with a thickness of $1.67 \mathrm{~m}$ and a shear wave velocity of $151.6 \mathrm{~m} / \mathrm{s}$ on top of significantly stiffer layers of soil (figure 1a).

(a)

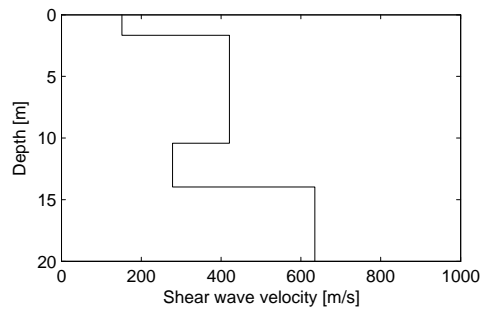

(b)

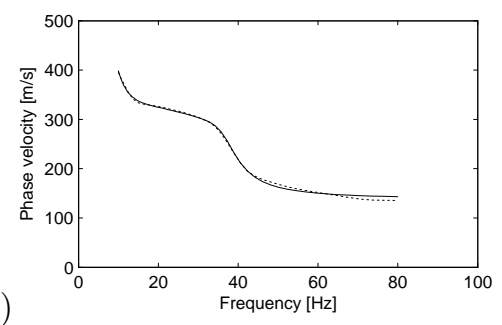

Figure 1: (a) Identified shear wave velocity profile and (b) corresponding theoretical dispersion curve (solid line) compared with the experimental curve (dashed line).

(a)

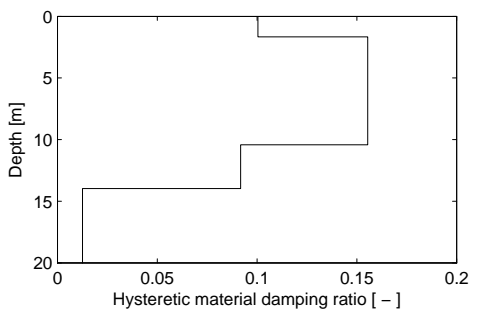

(b)

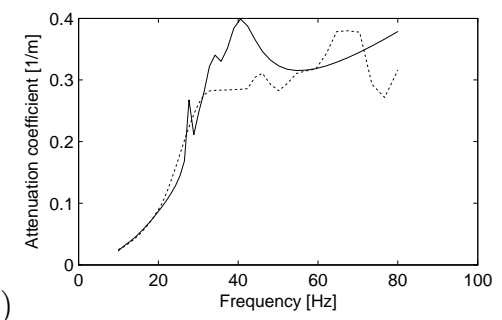

Figure 2: (a) Identified hysteretic material damping ratio and (b) corresponding theoretical attenuation curve (solid line) compared with the experimental curve (dashed line).

During the SASW test, the impact load was measured as well, allowing for the identification of the attenuation curve and, by fitting the computed to the identified attenuation curve (figure $2 \mathrm{~b}$ ), the hysteretic material damping ratio of the soil layers identified from the SASW test (figure 2a). The material damping ratio is assumed to be identical in shear and volumetric deformation. In the inversion, a soil density of $1850 \mathrm{~kg} / \mathrm{m}^{3}$ and Poisson's ratio of $1 / 3$ were assumed for all soil layers. The dynamic soil characteristics are summarized in table 1 . 
Table 1: Dynamic soil characteristics of the site at Saint-Epain.

\begin{tabular}{cccccc}
\hline Layer & $\begin{array}{c}d \\
{[\mathrm{~m}]}\end{array}$ & $\begin{array}{c}C_{s} \\
{[\mathrm{~m} / \mathrm{s}]}\end{array}$ & $\begin{array}{c}C_{p} \\
{[\mathrm{~m} / \mathrm{s}]}\end{array}$ & $\begin{array}{c}\rho \\
{\left[\mathrm{kg} / \mathrm{m}^{3}\right]}\end{array}$ & $\begin{array}{c}\beta \\
{[-]}\end{array}$ \\
\hline 1 & 1.67 & 151.6 & 303.2 & 1800 & 0.101 \\
2 & 8.76 & 420.8 & 841.6 & 1800 & 0.155 \\
3 & 3.54 & 278.4 & 556.8 & 1800 & 0.092 \\
4 & $\infty$ & 634.8 & 1269.6 & 1800 & 0.013 \\
\hline
\end{tabular}

The railway track on the conventional line Paris-Bordeaux is a classical ballasted track, with UIC60 rails supported by rail pads and fixed with clips on twin block concrete sleepers [33]. The track is supported by ballast with a thickness $h_{\mathrm{b}}$ of about $0.30 \mathrm{~m}$ on top of an embankment with a width $w_{\mathrm{e} 1}=5 \mathrm{~m}$ at the top, a width $w_{\mathrm{e} 2}=10 \mathrm{~m}$ at the soil's surface, and a height $h_{\mathrm{e}}=1.5 \mathrm{~m}$. The track-soil system has been modelled by coupling a 2.5D FE model for the track and the embankment to a 2.5D BE model for the soil $[22,34]$. Figure 3 shows a cross section of the model with a mesh of the $2.5 \mathrm{D}$ solid elements representing the ballast and embankment. The reader is referred to Lombaert et al. [7, 18] and François et al. [22] for more information on the model. In the following, the input parameters of the model are discussed.

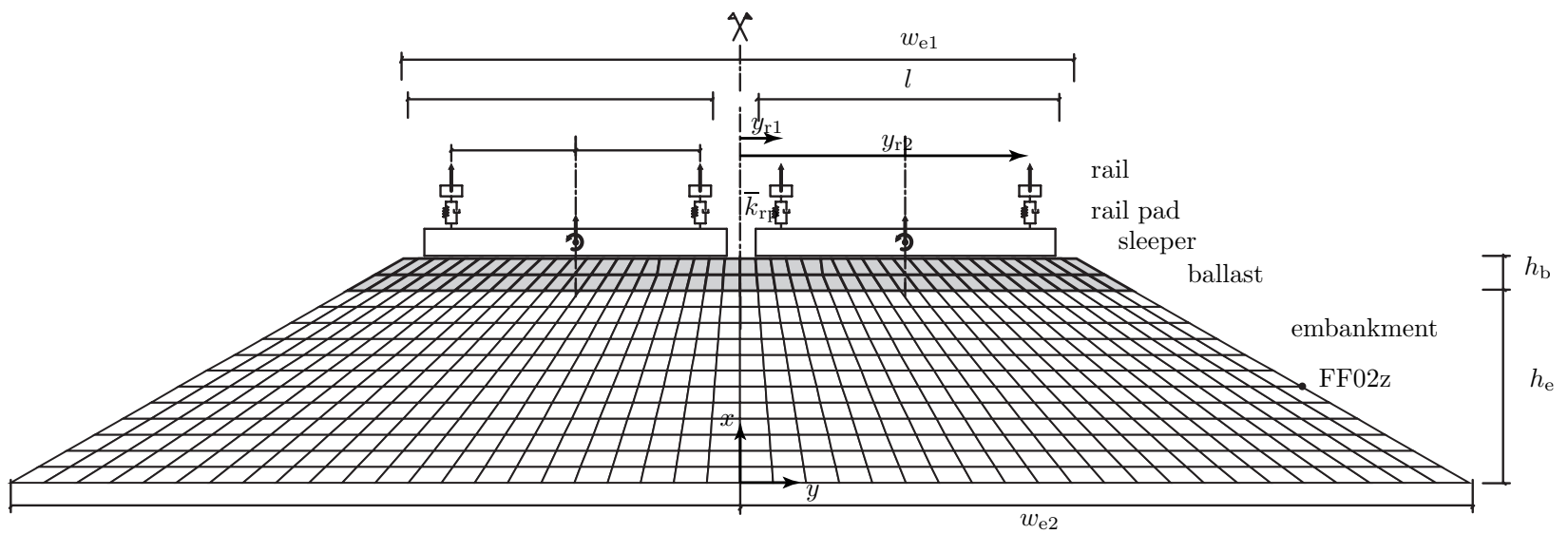

Figure 3: Cross section of model of the ballasted track on the embankment.

The rails are modelled as Euler-Bernoulli beams with a bending stiffness $E_{\mathrm{r}} I_{\mathrm{r}}=6.45 \times 10^{6} \mathrm{~N} / \mathrm{m}^{2}$ and a mass per unit length $\rho_{\mathrm{r}} A_{\mathrm{r}}=60.34 \mathrm{~kg} / \mathrm{m}$ for each rail. The positions of the rail are determined by $y_{\mathrm{r} 1}=1.145 \mathrm{~m}$ and $y_{\mathrm{r} 2}=2.580 \mathrm{~m}$, with $y_{\mathrm{r} 2}-y_{\mathrm{r} 1}$ equal to the track gauge $r_{\mathrm{d}}$. The internal energy dissipation in the rail is modelled by a loss factor $\eta_{\mathrm{r}}=0.05$. The rail pads are modelled as continuous spring-damper connections. The rail pad stiffness $k_{\mathrm{rp}}$ of a single rail pad and the sleeper spacing $d=0.6 \mathrm{~m}$ have been used to calculate an equivalent stiffness $\bar{k}_{\mathrm{rp}}=k_{\mathrm{rp}} / d=130 \times 10^{6} \mathrm{~N} / \mathrm{m}^{2}$. A loss factor $\eta_{\mathrm{rp}}=0.23$ is used for the rail pad. The concrete sleepers are assumed to be rigid in the plane of the track cross section and modelled as a uniformly distributed mass $\bar{m}_{\mathrm{sl}}=m_{\mathrm{sl}} / d$ of $417 \mathrm{~kg} / \mathrm{m}$. The sleeper's rotational inertia $\rho_{\mathrm{sl}} \bar{I}_{\mathrm{sl}}=\rho_{\mathrm{sl}} I_{\mathrm{sl}} / d$ is equal to $298 \mathrm{kgm}^{2} / \mathrm{m}$. The ballast bed is modelled as an elastic continuum, using $882.5 \mathrm{D}$ solid elements [22]. The Young's modulus of the ballast bed is computed from the vertical ballast stiffness per sleeper $k_{\mathrm{b}}=180 \times 10^{6} \mathrm{~N} / \mathrm{m}$ taking into account the support area of the twin block sleeper $2 l_{\mathrm{bl}} b_{\mathrm{bl}}=0.48 \mathrm{~m}^{2}$. This results in a ballast stiffness $K_{\mathrm{b}}=370 \times 10^{6} \mathrm{~N} / \mathrm{m}^{3}$ or a Young's modulus $E_{\mathrm{b}}=111 \times 10^{6} \mathrm{~N} / \mathrm{m}^{2}$. Additionally, a Poisson's ratio $\nu_{\mathrm{b}}=0.36$, density $\rho_{\mathrm{b}}=1550 \mathrm{~kg} / \mathrm{m}^{3}$, and loss factor $\eta_{\mathrm{b}}=1.00$ are assumed. The embankment is modelled as an elastic continuum using $5282.5 \mathrm{D}$ solid elements. The material properties of the embankment have been chosen equal to those of the top layer of soil (table 1 ). 


\subsection{The dynamic train characteristics}

At the site, ground vibrations have been measured during the passage of a single TGVA, a double TGVA, a Transport Express Régional (TER) commuter train of the type Autorail Grande Capacité (AGC), and two freight trains. In section 6 , the previously derived expressions for the ensemble average response quantities will be verified for the passage of a TGVA at a speed of $200 \mathrm{~km} / \mathrm{h}$ whereas predicted and measured ground vibrations during the passage of a TGVA and AGC TER commuter train at a speed of $200 \mathrm{~km} / \mathrm{h}$ and 150 $\mathrm{km} / \mathrm{h}$, respectively, will be compared in section 7 .

\section{The TGV Atlantique}

The TGVA is an articulated train with two traction cars and ten passenger cars. The two passenger cars adjacent to the traction cars share one bogie with the neighbouring passenger car, while the eight central passenger cars share both bogies with the neighbouring cars. The carriage length $L_{\mathrm{t}}$, the distance $L_{\mathrm{b}}$ between the bogies, the mass of the car body $M_{\mathrm{c}}$, and the pitching moment of inertia $J_{\mathrm{c}}$ of all carriages are given in table 2. The characteristics of the bogies are different for the traction cars and the passenger

Table 2: Geometrical and mass characteristics of the carriages of the TGVA.

\begin{tabular}{lccccc}
\hline & $\begin{array}{c}\text { Axles } \\
{[-]}\end{array}$ & $\begin{array}{c}L_{\mathrm{t}} \\
{[\mathrm{m}]}\end{array}$ & $\begin{array}{c}L_{\mathrm{b}} \\
{[\mathrm{m}]}\end{array}$ & $\begin{array}{c}M_{\mathrm{c}} \\
{[\mathrm{kg}]}\end{array}$ & $\begin{array}{c}J_{\mathrm{c}} \\
{\left[\mathrm{kgm}^{2}\right]}\end{array}$ \\
\hline 2 Traction cars & 4 & 22.15 & 14.00 & 55790 & $1.15 \times 10^{6}$ \\
2 Side cars & 3 & 21.84 & 18.70 & 24000 & $1.48 \times 10^{3}$ \\
8 Central cars & 2 & 18.70 & 18.70 & 24000 & $1.48 \times 10^{3}$ \\
\hline
\end{tabular}

cars. The bogie wheel base $L_{\mathrm{a}}$, the mass of the bogie $M_{\mathrm{b}}$, the pitching moment of inertia $J_{\mathrm{b}}$, and the unsprung mass $M_{\mathrm{u}}$ of the wheelsets for each type of car is given in table 3 .

Table 3: Geometrical and mass characteristics of the bogies of the TGVA.

\begin{tabular}{lcccc}
\hline & $\begin{array}{c}L_{\mathrm{a}} \\
{[\mathrm{m}]}\end{array}$ & $\begin{array}{c}M_{\mathrm{b}} \\
{[\mathrm{kg}]}\end{array}$ & $\begin{array}{c}J_{\mathrm{b}} \\
{\left[\mathrm{kgm}^{2}\right]}\end{array}$ & $\begin{array}{c}M_{\mathrm{u}} \\
{[\mathrm{m}]}\end{array}$ \\
\hline Traction car & 3.00 & 2380 & 1480 & 2048 \\
Passenger car & 3.00 & 3040 & 2680 & 2003 \\
\hline
\end{tabular}

Since the vehicle's primary and secondary suspension isolate the bogie and the vehicle body from the wheelset [35] at frequencies of more than a few Hertz, the motion of the car body can generally be disregarded in the calculation of the dynamic axle loads. A simplified vehicle model is therefore adopted that contains 4 degrees of freedom for each bogie: the vertical displacement and the rotation about the center of gravity of the bogie, and the vertical displacement of both wheelsets (figure 4). Table 4 summarizes the stiffness $k_{1}$ and damping $c_{1}$ of the primary suspension system, and the stiffness $k_{2}$ and damping $c_{2}$ of the secondary suspension.

Table 4: Dynamic characteristics of the primary and secondary suspension of the TGVA.

\begin{tabular}{lcccc}
\hline & $k_{1}$ & $c_{1}$ & $k_{2}$ & $c_{2}$ \\
& {$\left[\times 10^{6} \mathrm{~N} / \mathrm{m}\right]$} & {$\left[\times 10^{3} \mathrm{Ns} / \mathrm{m}\right]$} & {$\left[\times 10^{6} \mathrm{~N} / \mathrm{m}\right]$} & {$\left[\times 10^{3} \mathrm{Ns} / \mathrm{m}\right]$} \\
\hline Traction car & 2.45 & 20 & 2.45 & 40 \\
Passenger car & 1.40 & 10 & 0.82 & 48 \\
\hline
\end{tabular}




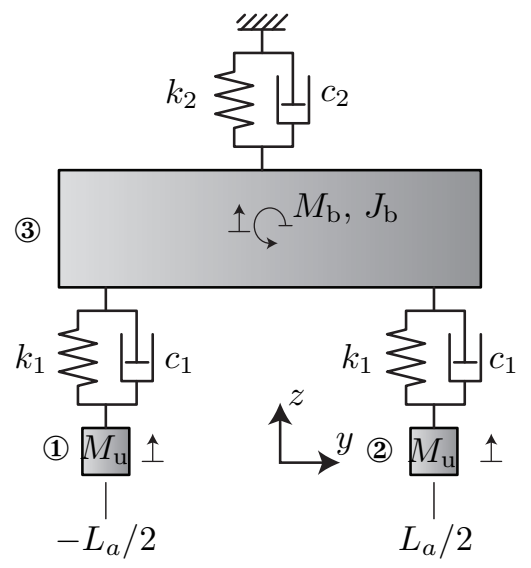

Figure 4: 4 degree of freedom bogie model

Based on the mass of the car body, the bogie mass, and the unsprung mass of the wheelsets, the total axle load is estimated as $169 \mathrm{kN}$ for the traction car, $113 \mathrm{kN}$ for the side cars, and $155 \mathrm{kN}$ for the central cars.

\section{The AGC TER commuter train}

The AGC TER commuter train is an articulated train with two traction cars and one central car. The distance $L_{\mathrm{b}}$ between the bogies and the mass of the car body $M_{\mathrm{c}}$ of the traction cars and the central car is given in table 5 .

Table 5: Geometrical and mass characteristics of the carriages of the AGC TER commuter train.

\begin{tabular}{lccc}
\hline & $\begin{array}{c}\text { Axles } \\
{[-]}\end{array}$ & $\begin{array}{c}L_{\mathrm{b}} \\
{[\mathrm{m}]}\end{array}$ & $\begin{array}{c}M_{\mathrm{c}} \\
{[\mathrm{kg}]}\end{array}$ \\
\hline 2 Traction cars & 3 & 17.20 & 50400 \\
1 Central car & 2 & 15.40 & 32600 \\
\hline
\end{tabular}

The traction cars have one traction bogie and share a coach bogie with the adjacent central car. The bogie wheel base $L_{\mathrm{a}}$, the mass of the bogie $M_{\mathrm{b}}$, the pitching moment of inertia $J_{\mathrm{b}}$, and the unsprung mass $M_{\mathrm{u}}$ of the wheelsets for each type of bogie are given in table 6 . The pitching moment of inertia $J_{\mathrm{b}}$ has been estimated from the bogie mass $M_{\mathrm{b}}$, assuming the bogie mass to be distributed as two point masses $M_{\mathrm{b}} / 2$ at a distance of $L_{\mathrm{a}} / 2$. Table 7 summarizes the stiffness and damping of the primary and secondary suspension system of the AGC TER commuter train. In the following, only the motion of the bogie and the wheelsets is taken into account as in the case of the TGVA. The train model therefore consists of a sequence of 4 bogies (figure 4).

Table 6: Geometrical and mass characteristics of the bogies of the AGC TER commuter train.

\begin{tabular}{lcccc}
\hline & $\begin{array}{c}L_{\mathrm{a}} \\
{[\mathrm{m}]}\end{array}$ & $\begin{array}{c}M_{\mathrm{b}} \\
{[\mathrm{kg}]}\end{array}$ & $\begin{array}{c}J_{\mathrm{b}} \\
{\left[\mathrm{kgm}^{2}\right]}\end{array}$ & $\begin{array}{c}M_{\mathrm{u}} \\
{[\mathrm{m}]}\end{array}$ \\
\hline Traction bogie & 3.00 & 9800 & 5500 & 2030 \\
Coach bogie & 3.00 & 5800 & 3300 & 2030 \\
\hline
\end{tabular}


Table 7: Dynamic characteristics of the primary and secondary suspension of the AGC TER commuter train.

\begin{tabular}{|c|c|c|c|c|}
\hline & $\begin{array}{c}k_{1} \\
{\left[\times 10^{6} \mathrm{~N} / \mathrm{m}\right]}\end{array}$ & $\begin{array}{c}c_{1} \\
{\left[\times 10^{3} \mathrm{Ns} / \mathrm{m}\right]}\end{array}$ & $\begin{array}{c}k_{2} \\
{\left[\times 10^{6} \mathrm{~N} / \mathrm{m}\right]}\end{array}$ & $\begin{array}{c}c_{2} \\
{\left[\times 10^{3} \mathrm{Ns} / \mathrm{m}\right]}\end{array}$ \\
\hline Traction bogie & 2.80 & 5 & 0.53 & 9 \\
\hline Coach bogie & 6.50 & 5 & 0.30 & 9 \\
\hline
\end{tabular}

Based on the mass of the car body, the bogie mass, and the unsprung mass of the wheelsets, the total axle load is estimated as $226 \mathrm{kN}$ for the traction cars and $208 \mathrm{kN}$ for the central car.

\subsection{Track unevenness}

The track unevenness at the site has been determined by means of a measurement trolley and a measurement coach [33]. A single-sided power spectral density function $\tilde{S}_{\mathrm{r} z z}\left(n_{y}\right)\left[\mathrm{m}^{3}\right]$ of the following type has been fitted to the data:

$$
\tilde{S}_{\mathrm{r} z z}\left(n_{y}\right)=\tilde{S}_{\mathrm{r} z z}\left(n_{y 0}\right)\left(\frac{n_{y}}{n_{y 0}}\right)^{-w}
$$

where $\tilde{S}_{\mathrm{r} z z}\left(n_{y 0}\right)$ is the reference value of the PSD at $n_{y 0}=1 /(2 \pi) \mathrm{m}^{-1}$ and $w$ is the exponent that determines the decrease of the PSD function with increasing cyclic wavenumber $n_{y}$. By fitting the PSD function to the experimental data in the range of wavelengths between $0.315 \mathrm{~m}$ and $3.15 \mathrm{~m}$, the coefficients $S\left(n_{y 0}\right)=$ $9.59 \times 10^{-7} \mathrm{~m}^{3} / \mathrm{rad}$ and $w=4.40$ were obtained. This fit is required to extrapolate the track unevenness beyond the measured range of wavelengths. For train speeds between $150 \mathrm{~km} / \mathrm{h}$ and $200 \mathrm{~km} / \mathrm{h}$ as considered next, the measured wavelength range covers excitation between 13.2 and $132 \mathrm{~Hz}$ and 17.6 and $176 \mathrm{~Hz}$, respectively. Dynamic axle loads outside this frequency range are therefore depending on the extrapolation, while ground vibration response is affected in a slightly wider frequency range because of the Doppler effect. This is important when considering the comparison between predicted and measured ground vibration in section 7 .

\section{Verification of the estimated variability of the free field response}

\subsection{Predicted free field velocity for 6 realizations of track unevenness}

In order to demonstrate the variability of the free field response, the dynamic axle loads have been computed for 6 realizations of track unevenness that have been generated from the PSD function in equation (45). The realizations are generated as a superposition of harmonic functions with random phase angles according to the spectral representation theorem [36, 37]:

$$
u_{\mathrm{r} z}(y)=\sum_{m=1}^{n} \sqrt{2 \tilde{S}_{\mathrm{r} z z}\left(k_{y m}\right) \Delta k_{y}} \cos \left(k_{y m} y-\theta_{m}\right)
$$

where $k_{y m}=m \Delta k_{y}$ is the wavenumber sampling, $\Delta k_{y}$ the wavenumber bin and $\theta_{m}$ are independent random phase angles uniformly distributed in the interval $[0,2 \pi]$. The realizations have a period $Y=2 \pi / \Delta k_{y}$ and are asymptotically Gaussian as $n$ tends to infinity and $\Delta k_{y}$ tends to zero for a fixed value of $k_{y}^{\max }=n \Delta k_{y}$. The wavenumber bin $\Delta k_{y}$ should be sufficiently small to avoid that the predicted ground vibration is affected by the periodicity of the generated track unevenness. The wavenumber range $\left[0, k_{y}^{\max }\right]$ is determined by the required frequency range of excitation. The dynamic axle loads are computed from the track unevenness using a compliance formulation that allows accounting for dynamic train-track interaction in a moving frame of reference [38].

Figure 5 shows the running RMS value and one-third octave band spectrum at $2 \mathrm{~m}, 12 \mathrm{~m}, 32 \mathrm{~m}, 56 \mathrm{~m}$, and $72 \mathrm{~m}$ from the outer rail of the track for the passage of a TGVA at a speed of $200 \mathrm{~km} / \mathrm{h}$ and the 6 realizations 


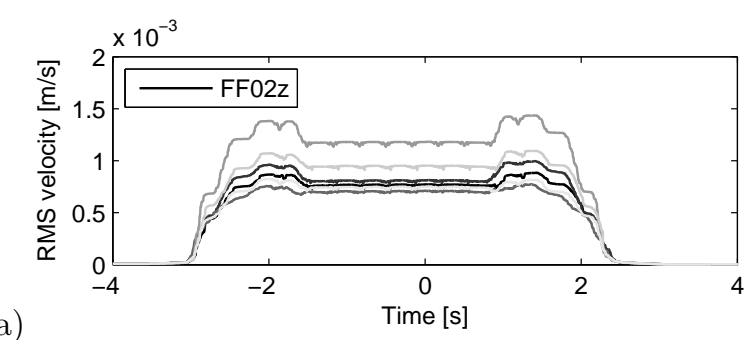

(a)

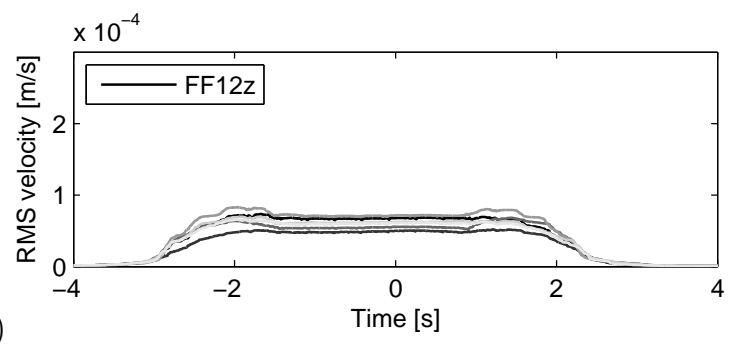

(b)

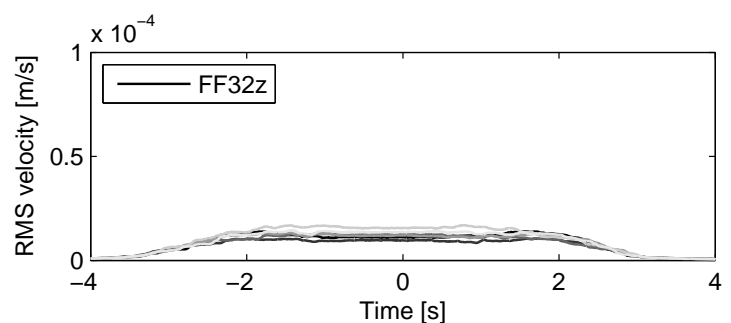

(c)

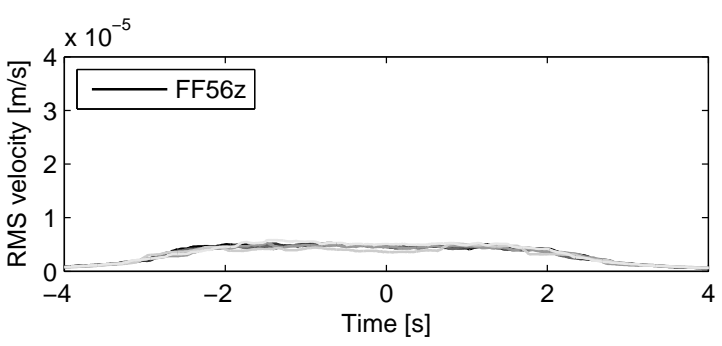

(d)

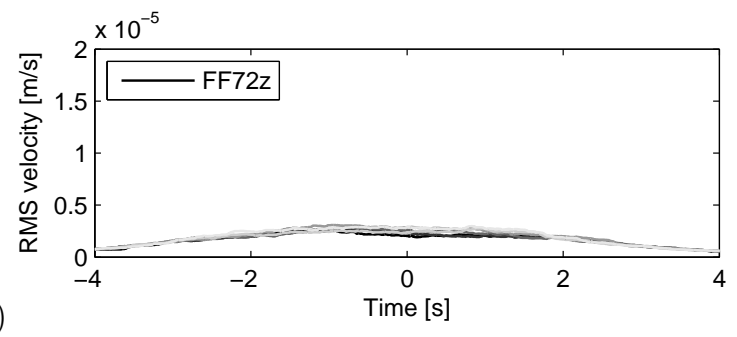

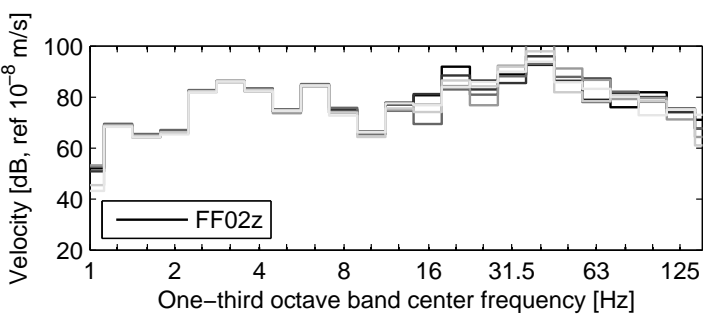

(f)

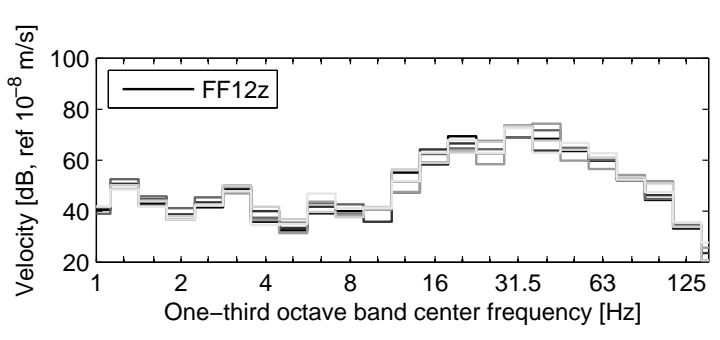

(g)

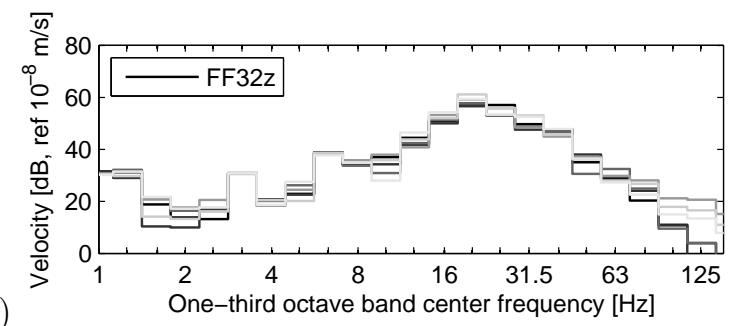

(h)

(i)
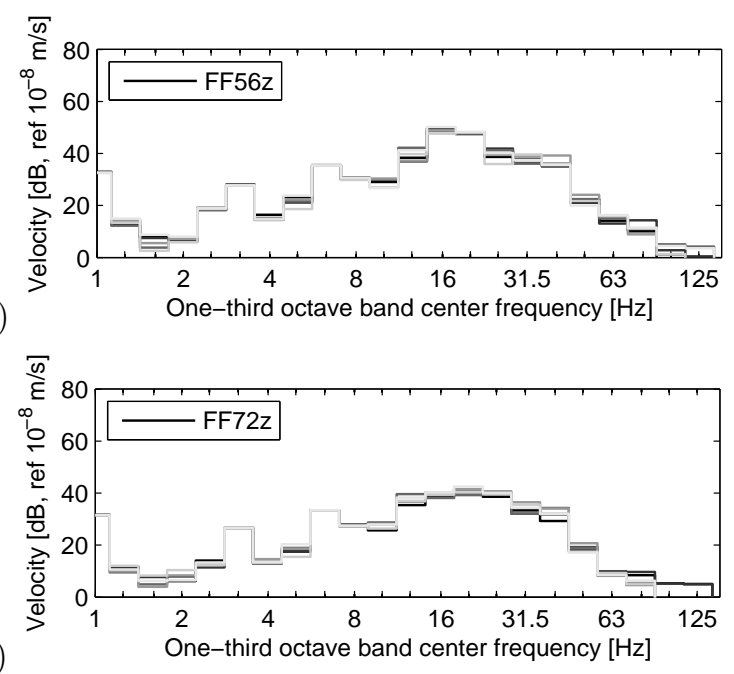

Figure 5: (a)-(e) Running RMS value and (f)-(j) one-third octave band spectrum of the predicted free field velocity at $2 \mathrm{~m}$, $12 \mathrm{~m}, 32 \mathrm{~m}, 56 \mathrm{~m}$ and $72 \mathrm{~m}$ from the outer rail during the passage of a TGVA at a speed of $200 \mathrm{~km} / \mathrm{h}$ for 6 realizations of track unevenness.

of track unevenness. Note that the point at $2 \mathrm{~m}$ from the outer rail is situated on the embankment. The running RMS value has been computed with a time window of $1 \mathrm{~s}$ as prescribed by the ISO 2631 standard [39]. The running RMS value increases during the approach of the train, reaches a stationary value while the train passes in front of the receiver point and than decreases again. Close to the track, the stationary vibration level is nearly constant for each of the 6 realizations and the difference between the 6 realizations 
does not change during the passage of the train. Further away from the track, the running RMS level of the response for a single realization of the track unevenness is time dependent and the difference between the 6 realizations shows more variability in time. This is due to the fact that, close to the track, the axles in the immediate vicinity of the receiver point have the largest contribution. The dynamic excitation is therefore determined by the unevenness on a relatively short stretch of track. Further away from the track, the response is determined by a larger number of axles and therefore depends on the unevenness on a longer stretch of track.

The one-third octave band spectra in figure 5 have been computed according to the German standard DIN 45672-2 [32] on a reference period $T_{2}$ during which the response is considered to be stationary. Close to the track, the response below $16 \mathrm{~Hz}$ is predominantly due to quasi-static excitation and therefore unaffected by differences in track unevenness. Further away from the track, the low frequency components are also determined by dynamic excitation and, therefore, different for the 6 realizations of track unevenness. In general, it can be concluded from figure 5 that a substantial variability is observed between the response for different realizations of track unevenness, with differences of up to $10 \mathrm{~dB}$ between the one-third octave band spectra. This confirms the need for statistical procedures that allow characterizing the response variability.

\subsection{Ensemble average of the response}

A Monte Carlo simulation has been performed to verify the proposed procedure for the assessment of the response variability. In the Monte Carlo simulation, the free field response was computed for 512 realizations of track unevenness. This number was sufficient for obtaining convergence of all results considered next. Whereas in the previous subsection the running RMS value of the vibration velocity was discussed for individual realizations of track unevenness, the ensemble average considered here is the mean square value across realizations of track unevenness. Figure 6 compares the time dependent RMS value found from the 512 realizations to the square root of the mean square response, given in equation (21) for the time domain response and equation (25) for the frequency domain response. Since the RMS value is not computed by averaging over a time window of $1 \mathrm{~s}$ as in figure 5, the passage of individual bogies can be recognized up to $32 \mathrm{~m}$ from the track. Figure 6 shows that an excellent agreement is obtained between both results.

Next, the calculation of the ensemble average running RMS value of the response according to equation (22) and the one-third octave band spectra according to equation (26) is verified. Figure 7 compares the resulting ensemble average values with the corresponding mean values computed from the 512 track unevenness realizations. The passage time $T_{\mathrm{p}}$ is calculated as the reference period $T_{2}$ of the German standard DIN 45672-2 [32] for the ensemble average running RMS value of the response. An excellent agreement between both results is generally obtained for these results as well. At larger distances from the track, the small difference between the ensemble average of the one-third octave band spectra estimated from equation (26) and the mean value of the 512 realizations is probably due to the selection of the stationary response part.

\subsection{Scatter of the response with respect to the ensemble average}

After the verification of the ensemble average response quantities, the scatter of the response is considered. In the present case where track unevenness is assumed to be a stationary Gaussian random field and the track geometry and properties are homogeneous along the track, the dynamic axle loads are stationary Gaussian random processes and the response at a fixed point in the free field is a non-stationary Gaussian random process. Equations (32) and (34) show how confidence bounds for the response at a particular time can be found from the instantaneous RMS value of the response (figure 6) and the CDF of the chi-square distribution. In case the contribution due to dynamic excitation dominates the total response, equation (32) holds and the $75 \%$ confidence bounds are given by 0.18 and 1.52 times the instantaneous RMS value of the response as shown before. Figure 8 compares the resulting scaled ensemble average values to the $12.5 \%$ and $87.5 \%$ quantiles computed from the 512 realizations. An excellent agreement is obtained between both results, validating the proposed procedure for the characterization of the response variability.

In a similar way, equations (42) and (43) show how confidence bounds are computed for the narrow band response spectrum based on the ensemble average (figure 6) and the CDF of the chi-square distribution. 


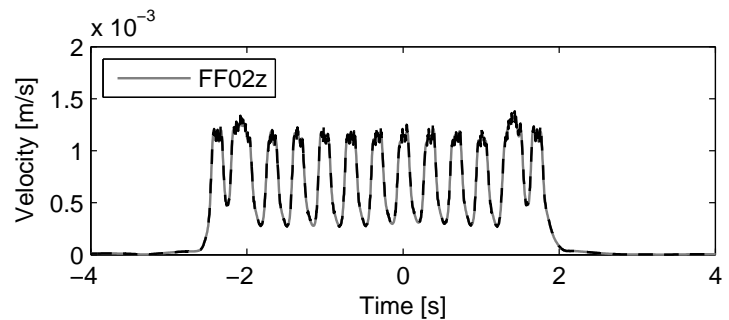

(a)
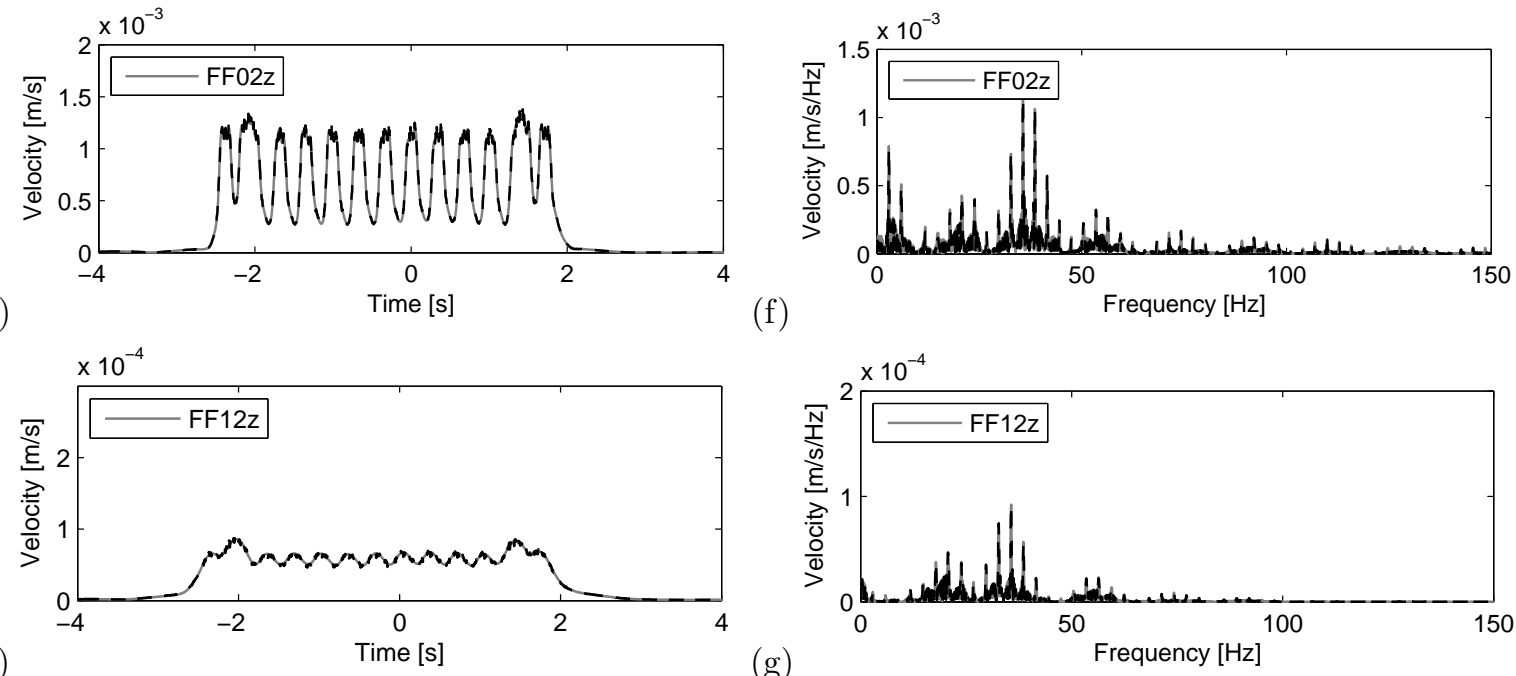

(b)

(g)


(c)
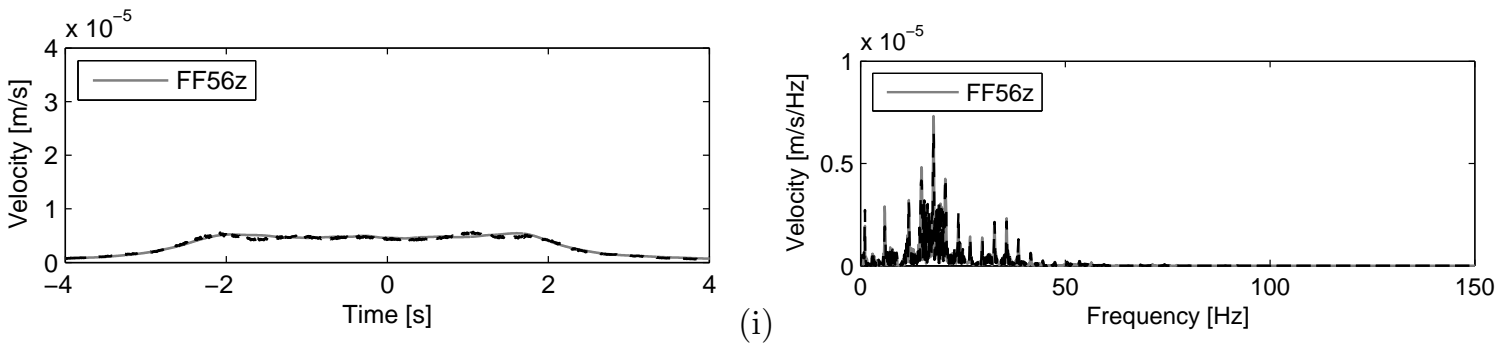

(d)


Figure 6: Root mean square value of (a)-(e) the time domain response and (f)-(j) the modulus of the narrow band spectrum of the free field velocity at $2 \mathrm{~m}, 12 \mathrm{~m}, 32 \mathrm{~m}, 56 \mathrm{~m}$ and $72 \mathrm{~m}$ from the outer rail during the passage of a TGVA at a speed of $200 \mathrm{~km} / \mathrm{h}$ computed by the statistical procedure (grey line) and estimated as the average value for 512 realizations (dashed black line).

Assuming again that the dynamic contribution dominates the response, equation (42) prevails and the 75 $\%$ confidence bounds are given by 0.134 and 1.44 times the ensemble average value. A comparison with the $12.5 \%$ and $87.5 \%$ quantiles of the narrow band spectra computed from the 512 realizations in figure 8 shows that a similar level of agreement is obtained in the frequency domain. 
(a)


(b)



(c)



(d)

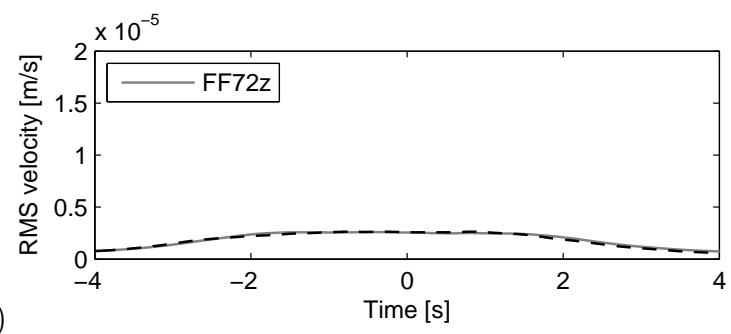

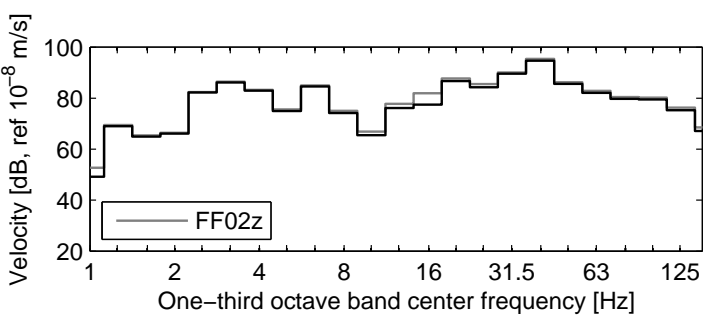

(f)



(g)

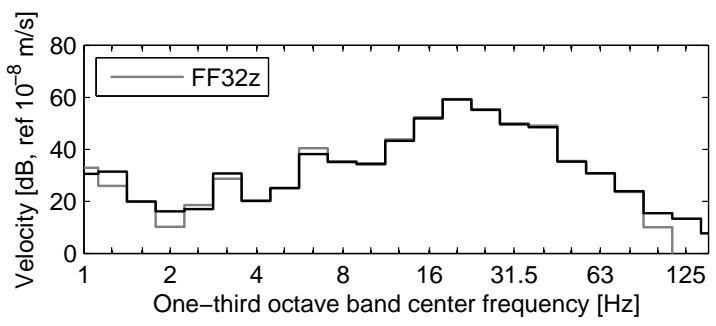

(h)

(i)



(j)

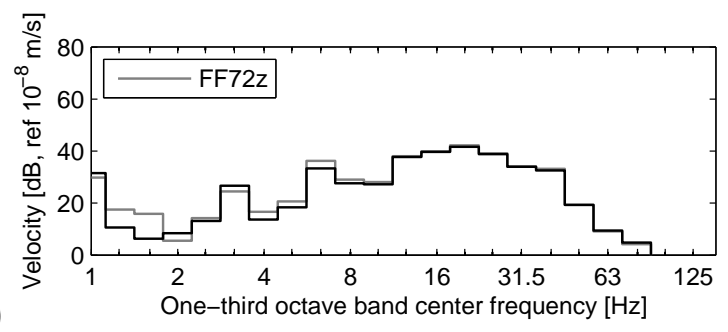

Figure 7: Ensemble average of (a)-(e) the running RMS value and (f)-(j) the one-third octave band spectrum of the free field velocity at $2 \mathrm{~m}, 12 \mathrm{~m}, 32 \mathrm{~m}, 56 \mathrm{~m}$ and $72 \mathrm{~m}$ from the outer rail during the passage of a TGVA at a speed of $200 \mathrm{~km} / \mathrm{h}$ computed by the statistical procedure (grey line) and estimated as the average value for 512 realizations (dashed black line).

As a final verification of the proposed statistical procedure, the variability of the running RMS value on a time window $T_{\mathrm{w}}$ of $1 \mathrm{~s}$ is estimated from the ensemble average response value (figure 7 ) in the same way as for the instantaneous response value (figure 8). As shown by equation (40), the variability in the running RMS value of the response also depends on the correlation in time within the window $T_{\mathrm{w}}$. When the same factors are applied to compute the confidence bounds from the ensemble average of the running RMS value 



(b)

$(\mathrm{g})$
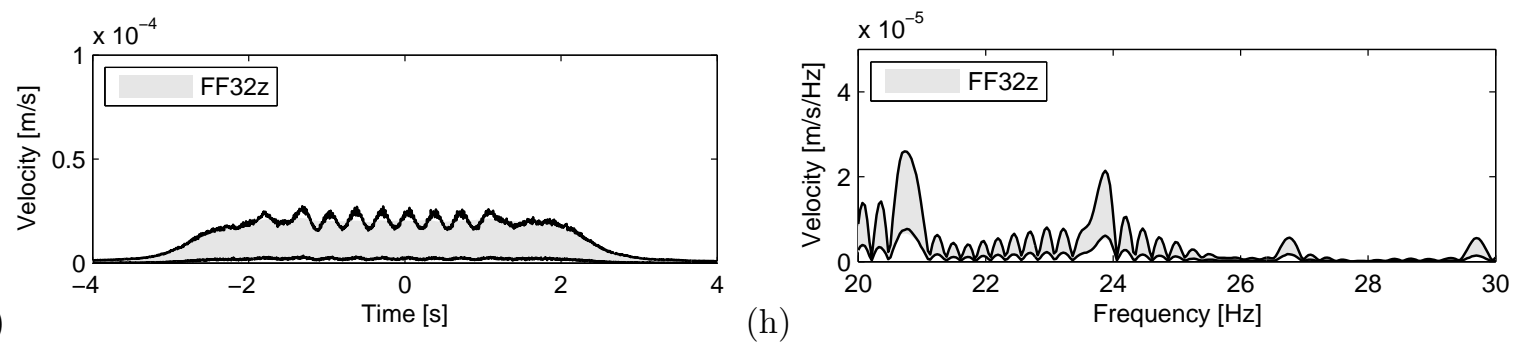

(c)

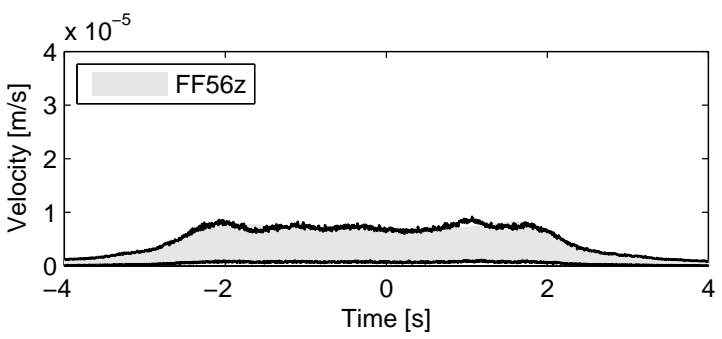

(d)

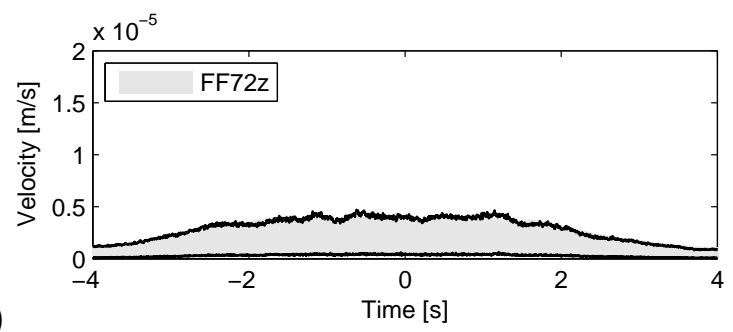

(h)



(i)

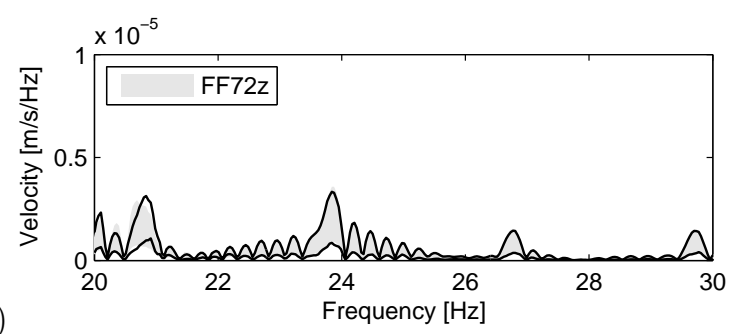

Figure 8: The $75 \%$ confidence region for (a)-(e) the absolute value and (f)-(j) the modulus of the narrow band spectrum of the free field velocity at $2 \mathrm{~m}, 12 \mathrm{~m}, 32 \mathrm{~m}, 56 \mathrm{~m}$ and $72 \mathrm{~m}$ from the outer rail during the passage of a TGVA at a speed of $200 \mathrm{~km} / \mathrm{h}$ computed by the statistical procedure (grey area) and upper and lower bounds estimated from the results of 512 realizations (black lines).

as for the instantaneous value, the response is assumed to be fully correlated within the time window $T_{\mathrm{w}}$, and a conservative estimate of the confidence bounds is obtained. This is clearly observed from figure 9 where these confidence bounds are compared to the $12.5 \%$ and $87.5 \%$ quantiles computed from the 512 realizations. 




(a)

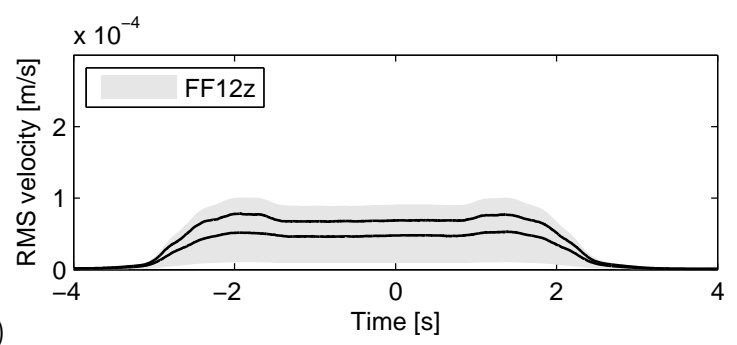

(b)

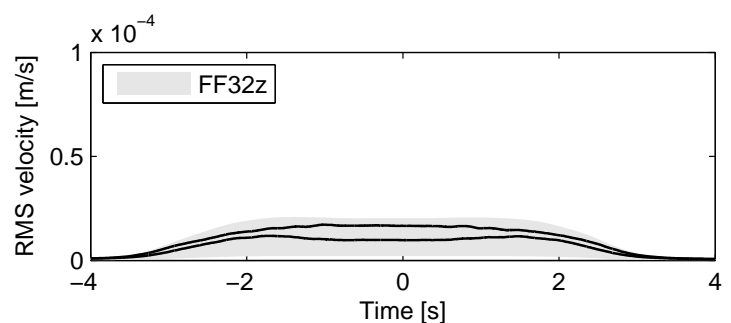

(c)

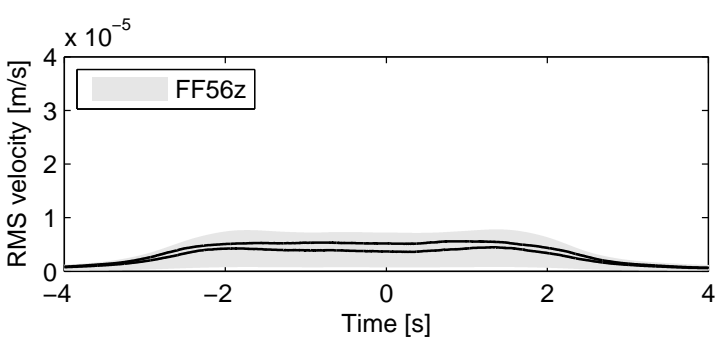

(d)



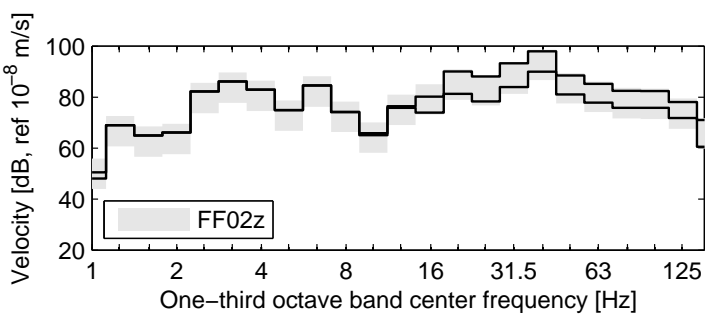

(f)

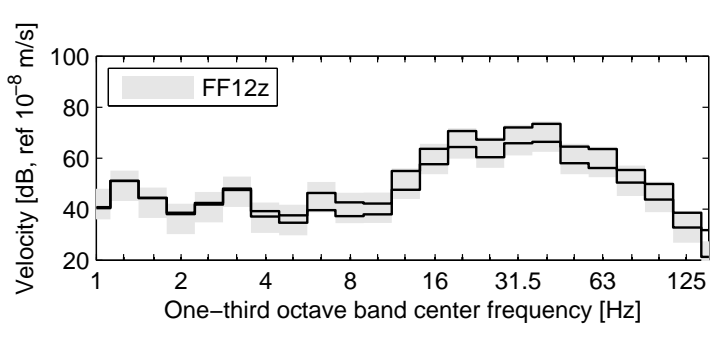

(g)



(h)

(i)
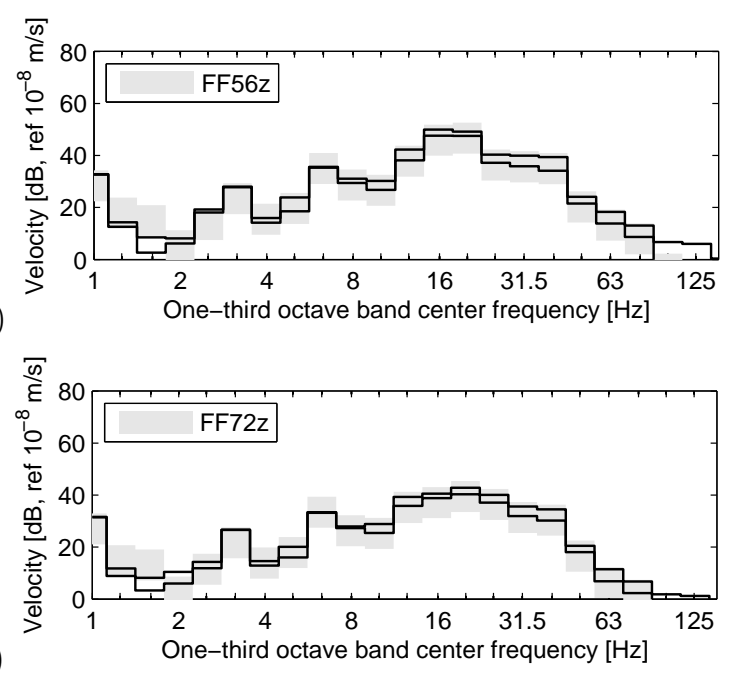

Figure 9: The $75 \%$ confidence region for (a)-(e) the running RMS value and (f)-(j) the one-third octave band spectrum of the free field velocity at $2 \mathrm{~m}, 12 \mathrm{~m}, 32 \mathrm{~m}, 56 \mathrm{~m}$ and $72 \mathrm{~m}$ from the outer rail during the passage of a TGVA at a speed of $200 \mathrm{~km} / \mathrm{h}$ computed by the statistical procedure (grey area) and upper and lower bounds estimated from the results of 512 realizations (black lines).

Figure 9 shows similar results for the one-third octave band spectra of the free field velocity. In the frequency range where the dynamic contribution dominates the response, the confidence bounds derived according to the procedure for the narrow band spectrum provide a conservative estimate of the $12.5 \%$ and $87.5 \%$ quantiles computed from the 512 realizations. At $2 \mathrm{~m}$ from the outer rail (figure 9f), the $12.5 \%$ 
and $87.5 \%$ quantiles coincide below $16 \mathrm{~Hz}$, where quasi-static excitation is most important, and separate at higher frequencies. In this case, the assumption of a dominating dynamic response contribution no longer holds.

Although a significant degree of overestimation is present in the confidence bounds of both the time and frequency domain RMS values, the latter are still very useful to assess the discrepancy between numerical predictions and measurements of ground borne vibration. This is illustrated in the next section where the predicted and measured free field velocity are compared for the passage of a TGVA at $200 \mathrm{~km} / \mathrm{h}$ and an AGC TER commuter train at $150 \mathrm{~km} / \mathrm{h}$.

\section{Comparison of measured and predicted free field velocity}

\subsection{Passage of a TGVA at a speed of $200 \mathrm{~km} / \mathrm{h}$}

On the site at Saint-Epain, the accelerations at $2 \mathrm{~m}, 12 \mathrm{~m}, 32 \mathrm{~m}, 56 \mathrm{~m}$, and $72 \mathrm{~m}$ from the outer rail have been measured at a sampling frequency of $4096 \mathrm{~Hz}$. A Chebyshev window in the time domain has been applied to eliminate noise measured before and after the train passage. The free field velocities have been obtained by integrating the accelerations by means of a trapezium rule. A third-order Chebyshev filter has been used to eliminate the DC-component and to suppress drifting of the integrated signals. A high-pass frequency of $2 \mathrm{~Hz}$ has been used at $2 \mathrm{~m}$ and was increased to $3 \mathrm{~Hz}$ further away from the track.

The statistical procedure for characterizing the response variability is now used to assess the difference between predicted and measured results of the free field velocity during the passage of a TGVA at a speed of $200 \mathrm{~km} / \mathrm{h}$. Figure 10 compares the predicted ensemble average of the running RMS value and one-third octave band spectra of the free field velocity to values measured by SNCF. On the figures, the conservative estimate of the $75 \%$ confidence bounds, previously shown in figure 9 , is included as well.

At $2 \mathrm{~m}$ from the outer rail (figures 10a and 10f), which is still on the embankment, the response is considerably overestimated up to $39 \mathrm{~Hz}$. In most of this frequency range, quasi-static excitation dominates over dynamic excitation as can be observed from the results for the 6 realizations of track unevenness in figure 5f. The confidence bounds characterize the scatter of the dynamic response contribution and are therefore only meaningful for assessing the discrepancy between the predicted and measured free field velocity at higher frequencies. The overestimation in the low frequency range is therefore mostly due to the predicted quasi-static response, which depends on the static train loads, and the dynamic track and soil characteristics. The results suggest that the track and soil below the track are much stiffer than assumed in the calculations. It is not unlikely that, due to the repeated passage of trains, the soil below the track was densified and is therefore much stiffer than the soil next to the track where the SASW test has been performed. This was confirmed by additional calculations, not shown here for the sake of brevity, where the stiffness of the embankment and the top layer of soil were increased to the value of the stiffness of the second layer of soil (table 1).

For all receivers located between $12 \mathrm{~m}$ and $72 \mathrm{~m}$ from the track, a reasonable agreement is found in the one-third octave band spectra between $2 \mathrm{~Hz}$ and $39 \mathrm{~Hz}$. At these receivers, dynamic excitation dominates over quasi-static excitation except at very low frequencies (figure 5). The agreement at frequencies above $39 \mathrm{~Hz}$ is relatively good at $12 \mathrm{~m}$ from the track but deteriorates further away from the track, where the measured response is significantly larger than the upper limit of the range delimited by the confidence bounds of the predicted response. It is therefore highly unlikely that the discrepancy is due to the lack of information regarding track unevenness. The increased underestimation in this frequency range leads to an underestimation of the running RMS value of the vibration velocity at $56 \mathrm{~m}$ and $72 \mathrm{~m}$ from the track.

One of the reasons for the underestimation at higher frequencies may be the fact that parametric excitation due to periodic sleeper support is not accounted for. For a train speed of $200 \mathrm{~km} / \mathrm{h}$ and a sleeper spacing of $0.6 \mathrm{~m}$, the fundamental sleeper passing frequency $f_{\mathrm{sl}}$ is $92.6 \mathrm{~Hz}$, and the corresponding parametric excitation will contribute to ground vibration in a (limited) frequency range around $f_{\text {sl }}$ because of the Doppler effect. It is not easy to pinpoint other reasons for the mismatch between predictions and measurements. Uncertainties in the estimated parameters of the rolling stock, track, and soil play a role, as well as inaccuracies in the estimation of the PSD function of the track unevenness and its extrapolation beyond 
(a)


(b)

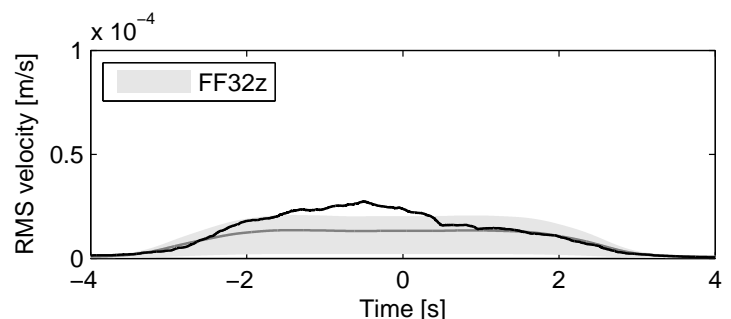

(c)

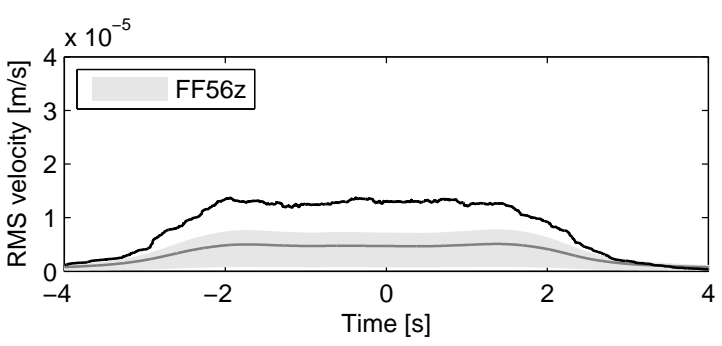

(d)

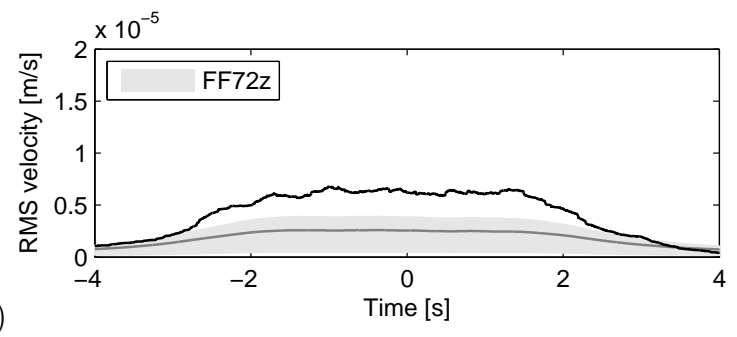



(f)



(g)

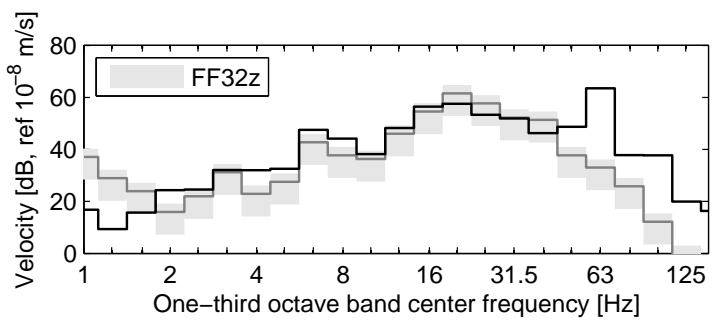

(h)

(i)
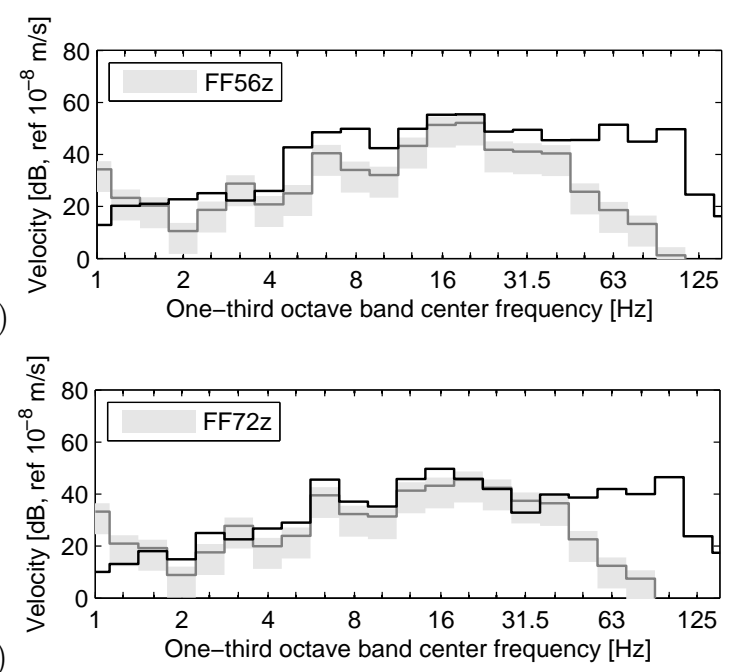

Figure 10: Predicted ensemble average (dark grey line) and measured (black line) (a)-(e) running RMS value and (f)-(j) onethird octave band spectrum of the free field velocity at $2 \mathrm{~m}, 12 \mathrm{~m}, 32 \mathrm{~m}, 56 \mathrm{~m}$ and $72 \mathrm{~m}$ from the outer rail during the passage of a TGVA at a speed of $200 \mathrm{~km} / \mathrm{h}$. Superimposed is the $75 \%$ confidence region for the predicted free field velocity (grey area).

the measured range of wavelengths. Discrepancies are probably also due to model uncertainties, however, as the $2.5 \mathrm{D}$ approach where track and soil geometry properties are assumed homogeneous along the track, necessitates significant simplifications of the problem geometry. Comparing the measured free field response above $63 \mathrm{~Hz}$ at $32 \mathrm{~m}, 56 \mathrm{~m}$, and $72 \mathrm{~m}$ from the track also reveals, however, that in this frequency range, the measured response is almost unattenuated. This suggests that this part of the response may be affected by 
high frequency noise.

7.2. Passage of the AGC TER commuter train at a speed of $150 \mathrm{~km} / \mathrm{h}$

(a)


(b)

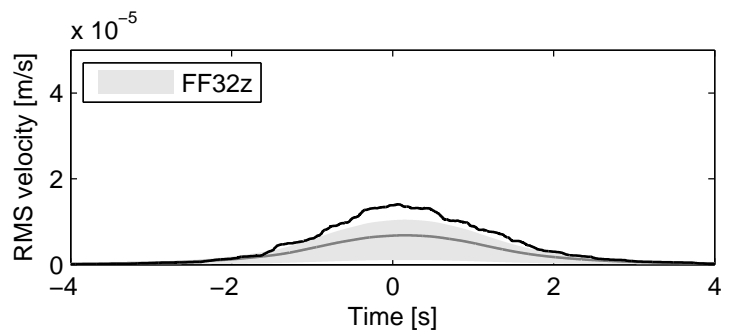

(c)



(d)



(f)
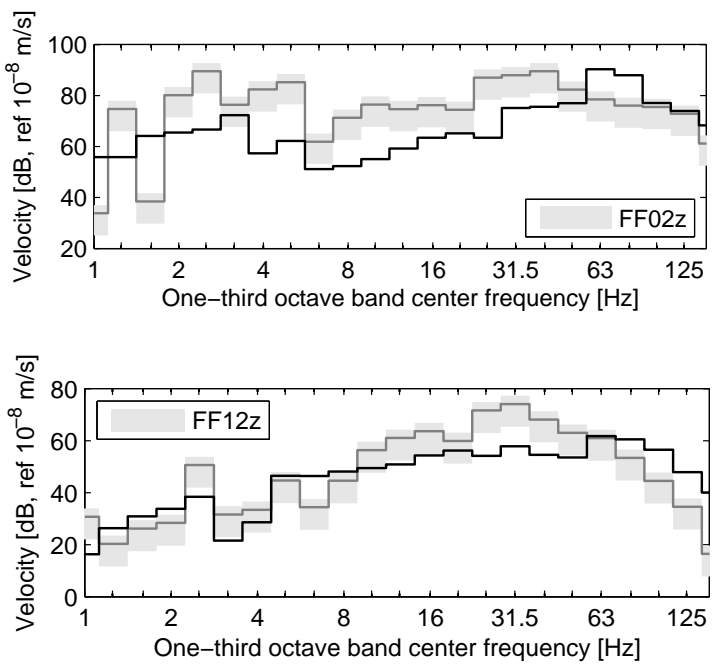

(g)

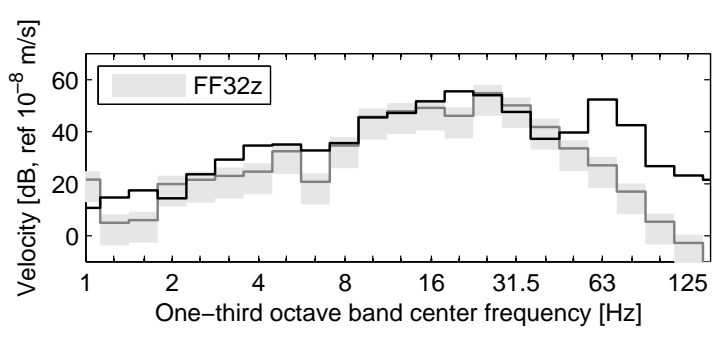

(i)

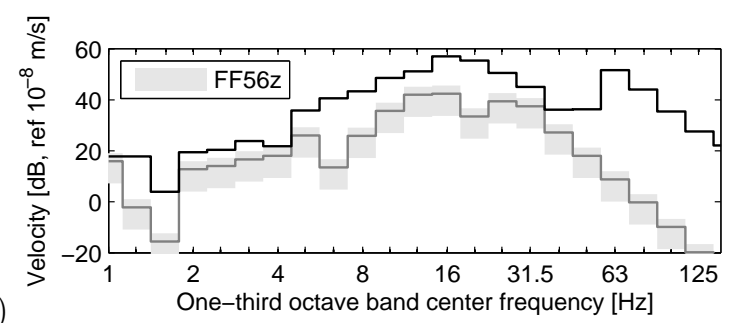

(j)

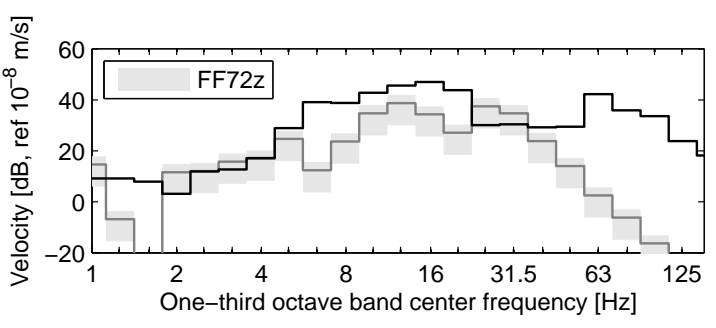

Figure 11: Predicted ensemble average for the updated (thick grey line) and original value (thin grey line) of the material damping ratio and measured (black line) (a)-(e) running RMS value and (f)-(j) one-third octave band spectrum of the free field velocity at $2 \mathrm{~m}, 12 \mathrm{~m}, 32 \mathrm{~m}, 56 \mathrm{~m}$ and $72 \mathrm{~m}$ from the outer rail during the passage of the AGC TER at a speed of $150 \mathrm{~km} / \mathrm{h}$. Superimposed is the $75 \%$ confidence region for the updated value of the material damping ratio (grey area). 
Figure 11 compares the predicted ensemble average of the running RMS value and one-third octave band spectra of the free field velocity to values measured by SNCF for the passage of the AGC TER train at a speed of $150 \mathrm{~km} / \mathrm{h}$. Similar observations can be made as for the passage of the TGVA in figure 10 . At $2 \mathrm{~m}$ from the outer rail, the response is significantly overestimated up to $39 \mathrm{~Hz}$, probably due to the fact that the embankment and soil below the track are much stiffer than assumed in the model. At distances of 12 $\mathrm{m}$ from the track and more, the response is reasonably well predicted below $39 \mathrm{~Hz}$. At higher frequencies, it is increasingly underestimated for larger distances from the track. This may be partly explained by the fact that parametric excitation, with the sleeper passage frequency now at $69 \mathrm{~Hz}$, is not accounted for. The measured free field response again shows a very small attenuation with distance above $63 \mathrm{~Hz}$, suggesting that the mismatch is also due to noise in the measured results.

\section{Conclusion}

In this paper, a statistical procedure has been presented that allows quantifying the variability in ground vibration predictions that arises from the statistical characterization of the track unevenness by a PSD function. The procedure has been derived assuming the dynamic axle loads to be stationary random processes and the track geometry to be homogeneous along the track. In this case, the ensemble average of the response, that represents the mean value for different realizations of the dynamic axle loads or, similarly, different realizations of track unevenness, can be computed from the PSD function of the dynamic axle loads. From the ensemble average of the instantaneous value and the narrow band spectrum of the response, the ensemble average of other response quantities can be computed such as running RMS values on a fixed time window or RMS values in one-third octave bands.

The characterization of the scatter with respect to the ensemble average values requires additional assumptions on the random processes that represent the dynamic axle loads. When the dynamic axle loads are stationary Gaussian random processes, the response at a fixed point in the free field is a nonstationary Gaussian random process. In this case, confidence bounds for the instantaneous response in the time domain and the narrow band spectrum can be calculated from the ensemble average value and the CDF of the chi-square distribution. In a similar way, a conservative estimate can be obtained for the confidence bounds for the running RMS values and one-third octave band spectra of the response.

The procedure has been successfully verified by means of Monte Carlo simulations and used to assess the discrepancy between predicted and measured values of the free field response in a case study. Even when it is hard to determine the exact reasons for the mismatch between predictions and measurements, the procedure allows assessing an important source of uncertainty in the computations.

\section{Acknowledgements}

The authors would like to thank the Innovation and Research Department of SNCF (Société Nationale des Chemins de fer Français) for providing the data of the case study.

The second author would like to thank the Spanish Ministry of Economy and Competitiveness (Ministerio de Economía y Competitividad) for their financial support under the research project BIA2010-14843.

The third author is a postdoctoral fellow of the Research Foundation - Flanders. The financial support is gratefully acknowledged.

\section{References}

[1] D.J. Thompson. Railway noise and vibration: mechanisms, modelling, and means of control. Elsevier, 2009.

[2] D. Aubry, D. Clouteau, and G. Bonnet. Modelling of wave propagation due to fixed or mobile dynamic sources. In N. Chouw and G. Schmid, editors, Workshop Wave '94, Wave propagation and Reduction of Vibrations, pages 109-121, Ruhr Universität Bochum, Germany, December 1994.

[3] A.V. Metrikine, S.N. Verichev, and J. Blauwendraad. Stability of a two-mass oscillator moving on a beam supported by a visco-elastic half-space. International Journal of Solids and Structures, 42:1187-1207, 2005.

[4] X. Sheng, C.J.C. Jones, and M. Petyt. Ground vibration generated by a harmonic load acting on a railway track. Journal of Sound and Vibration, 225(1):3-28, 1999. 
[5] X. Sheng, C.J.C. Jones, and M. Petyt. Ground vibration generated by a load moving along a railway track. Journal of Sound and Vibration, 228(1):129-156, 1999.

[6] L. Auersch. The excitation of ground vibration by rail traffic: theory of vehicle-track-soil interaction and measurements on high-speed lines. Journal of Sound and Vibration, 284(1-2):103-132, 2005.

[7] G. Lombaert, G. Degrande, J. Kogut, and S. François. The experimental validation of a numerical model for the prediction of railway induced vibrations. Journal of Sound and Vibration, 297(3-5):512-535, 2006.

[8] X. Sheng, C.J.C. Jones, and D.J. Thompson. Prediction of ground vibration from trains using the wavenumber finite and boundary element methods. Journal of Sound and Vibration, 293:575-586, 2006.

[9] J.A. Forrest and H.E.M. Hunt. A three-dimensional tunnel model for calculation of train-induced ground vibration. Journal of Sound and Vibration, 294:678-705, 2006.

[10] M.F.M. Hussein and H.E.M. Hunt. A numerical model for calculating vibration from a railway tunnel embedded in a full-space. Journal of Sound and Vibration, 305:401-431, 2007.

[11] L. Andersen and C.J.C. Jones. Coupled boundary and finite element analysis of vibration from railway tunnels - a comparison of two- and three-dimensional models. Journal of Sound and Vibration, 293(3-5):611-625, 2006.

[12] J.A. Forrest and H.E.M. Hunt. Ground vibration generated by trains in underground tunnels. Journal of Sound and Vibration, 294:706-736, 2006.

13] X. Sheng, C.J.C. Jones, and D.J. Thompson. A theoretical model for ground vibration from trains generated by vertical track irregularities. Journal of Sound and Vibration, 272(3-5):937-965, 2004.

14] G. Degrande, D. Clouteau, R. Othman, M. Arnst, H. Chebli, R. Klein, P. Chatterjee, and B. Janssens. A numerical model for ground-borne vibrations from underground railway traffic based on a periodic finite element - boundary element formulation. Journal of Sound and Vibration, 293(3-5):645-666, 2006.

[15] ORE. Question C116: Wechselwirkung zwischen Fahrzeugen und gleis, Bericht Nr. 1: Spektrale Dichte der Unregelmässigkeiten in der Gleislage. Technical report, Office for Research and Experiments of the International Union of Railways, Utrecht, NL, 1971.

[16] H. Braun and T. Hellenbroich. Messergebnisse von Strassenunebenheiten. VDI Berichte, 877:47-80, 1991.

[17] C. Esveld. Modern railway track. Second Edition. MRT-Productions, Zaltbommel, 2001.

[18] G. Lombaert and G. Degrande. Ground-borne vibration due to static and dynamic axle loads of InterCity and high speed trains. Journal of Sound and Vibration, 319(3-5):1036-1066, 2009.

[19] D.E. Newland. An introduction to random vibrations, spectral \& wavelet analysis. Longman Scientific \& Technical, Essex, England, 1994.

[20] F. Lu, Q. Gaoa, J.H. Lin, and F.W. Williams. Non-stationary random ground vibration due to loads moving along a railway track. Journal of Sound and Vibration, 298:30-42, 2006.

[21] G. Lombaert, G. Degrande, and D. Clouteau. Numerical modelling of free field traffic induced vibrations. Soil Dynamics and Earthquake Engineering, 19(7):473-488, 2000.

[22] S. François, M. Schevenels, G. Lombaert, P. Galvín, and G. Degrande. A 2.5D coupled FE-BE methodology for the dynamic interaction between longitudinally invariant structures and a layered halfspace. Computer Methods in Applied Mechanics and Engineering, 199(23-24):1536-1548, 2010.

[23] International Organization for Standardization. ISO 2631-1:1985: Evaluation of human body exposure to whole-body vibration - Part 1: General requirements, 1985.

[24] G. Degrande and G. Lombaert. An efficient formulation of Krylov's prediction model for train induced vibrations based on the dynamic reciprocity theorem. Journal of the Acoustical Society of America, 110(3):1379-1390, 2001.

[25] L. Auersch. Ground vibration due to railway traffic - The calculation of the effects of moving static loads and their experimental verification. Journal of Sound and Vibration, 293:599-610, 2006.

[26] H.E.M. Hunt. Modelling of road vehicles for calculation of traffic-induced ground vibrations. Journal of Sound and Vibration, 144(1):41-51, 1991.

[27] T.X. Wu and D.J. Thompson. Vibration analysis of railway track with multiple wheels on the rail. Journal of Sound and Vibration, 239(1):69-97, 2001.

[28] H. Verbraken, G. Lombaert, and G. Degrande. Verification of an empirical prediction method for railway induced vibrations by means of numerical simulations. Journal of Sound and Vibration, 330(8):1692-1703, 2011.

[29] International Organization for Standardization. ISO 2631-2:1989: Evaluation of human body exposure to whole-body vibration - Part 2: Continuous and shock-induced vibration in buildings (1 to $80 \mathrm{~Hz}$ ), 1989.

[30] C.G. Gordon. Generic criteria for vibration-sensitive equipment. In Proceedings of the SPIE Conference on Vibration Control and Metrology, volume 1619, pages 71-85, San Jose, California, USA, November 1991.

[31] H. Amick. On generic vibration criteria for advanced technology facilities. Journal of the Institute of Environmental Sciences, 40:35-44, 1997.

[32] Deutsches Institut für Normung. DIN 45672 Teil 2: Schwingungsmessungen in der Umgebung von Schienenverkehrswegen: Auswerteverfahren, 1995.

[33] E. Bongini and F. Poisson. Ground vibrations simulation cases parameters. Technical report, SNCF, France, 2009.

[34] P. Galvín, S. François, M. Schevenels, E. Bongini, G. Degrande, and G. Lombaert. A 2.5D coupled FE-BE model for the prediction of railway induced vibrations. Soil Dynamics and Earthquake Engineering, 30(12):1500-1512, 2010.

[35] K. Knothe and S.L. Grassie. Modelling of railway track and vehicle/track interaction at high frequencies. Vehicle Systems Dynamics, 22: 209-262, 1993.

[36] M. Shinozuka and C. Jan. Digital simulation of random processes and its application. Journal of Sound and Vibration, 25(1):111-128, 1972.

[37] M. Shinozuka and G. Deodatis. Simulation of stochastic processes by spectral representation. Applied Mechanics Reviews, 
44(4):191-204, 1991

[38] D. Clouteau, G. Degrande, and G. Lombaert. Numerical modelling of traffic induced vibrations. Meccanica, 36(4):401-420, 2001.

[39] International Organization for Standardization. ISO 2631-2:1999: Mechanical vibration and shock - Evaluation of human exposure to whole-body vibration - Part 2: Vibration in buildings (1 to $80 \mathrm{~Hz}$ ), 1999. 